\title{
Separation Axioms in Soft Tritopological Spaces with Respect to Ordinary Points
}

\author{
Asmhan Flieh Hassan \\ Department of Mathematics, Faculty of education for \\ girls, University of Kufa, Iraq
}

\author{
Ali Hussein Abaas \\ Department of Mathematics, Faculty of education for \\ girls, University of Kufa, Iraq
}

\begin{abstract}
In the present paper the definitions of separation axioms in soft -tritopological spaces are introduced dependent to the soft- $\delta^{*}$-open set and their basic properties are investigated with respect to ordinary points. That is, the soft $-\delta^{*}-\mathrm{T}_{\tilde{i}} ;(\tilde{i}=0,1,2,3,4)$ spaces and notions of soft $-\delta^{*}$-normal and soft $-\delta^{*}$-regular spaces are discussed in detail, also we introduce some theorems shows how one of the soft-spaces implies the others with the help of an examples it is established that the converse does not hold.
\end{abstract}

\section{General Terms}

2010 Mathematics Subject Classification: 54E55, 54D10.

\section{Keywords}

soft -tritopological space, soft $-\delta^{*}$ - open set, soft $-\delta^{*}-\mathrm{T}_{\hat{i}} ;(\hat{i}=0,1,2,3,4)$ space, soft $-\delta^{*}$-regular space, soft $-\delta^{*}$-normal space.

\section{INTRODUCTION}

The soft -theory was first initiated by Molodtsov [1] in 1999 as a mathematical tool for uncertain objects. In $[1,2]$, the researcher successfully applied the theory in several directions, like operations research, probability, theory of measurement, and others. To develop soft-set theory, the operations of the soft-sets are defined in [3].

A topology on a soft-sets, called "soft-topology", and its related properties which presented in 2011 by M Shabir \& M. Naz, [4] , Cagman et. al. [5], and they introduced the foundations of the theory of soft-topological spaces. A bitopology on a soft-sets, called 'soft-bitopology', and its related properties which presented by Ittanagi [6] in 2014, and he introduced the foundations of the theory of soft-bitopological spaces.

Furthermore, the theory of 'soft-tritopology' was first initiated by Asmhan [7] in 2017, and she was presented the foundations and its related properties. And in 2019 she was first initiated the theory of fuzzy - soft -tritopology [8]. In 2019, the Italian researcher Giorgio [9] introduced the theory of 'soft $-N$-topology', and study it in details.

Separation axioms in soft-topology are among the most interesting notions. Various generalizations of separation axioms have been studied for generalized soft-topological spaces, such as soft-bitopological. It is very interesting to see that when classical soft-notions are replaced by new generalized soft-notions. D. N. Georgiou et. al [10], A.
Kandil et. al. [11], El-Sheikh et. al. [12] and Shabir et. al [4], studied and defined some separation axioms in soft-topological spaces using (ordinary) points. A. M. Khattak et. al. [13] introduced soft-b-separation axioms in soft-bitopological spaces and discussed it with respect to ordinary and soft-points. And Ittanagi [6] introduced some types of soft-separation axioms in soft-bitopological spaces with respect to ordinary points. Also, The concept of pair-wise soft-separation axioms for bi-soft-topological spaces studied by M. Naz et. al. in [14].

In this paper, we define and explore several properties of soft $-\delta^{*}-\mathrm{T}_{\mathrm{i}} ; \quad(\quad \mathrm{i}=0,1,2,3,4)$ space, soft $-\delta^{*}$-regular space, soft $-\delta^{*}-$ normal space. All these axioms are defined using ordinary points. We also discuss some properties and obtained some results. We hope that these results will be useful for the future works on soft-tritopology to carry out general framework for some practical applications, such as medical, uncertainties problems and biomathematics.

Now, our motivation in the present paper is to state and continue the foundations of the soft-tritopological spaces theory. For more exactly, to define and study the sof $t-\delta^{*}$-separation axioms (based on soft $-\delta^{*}-$ open set) w.r.t. ordinary points and study the main properties for these soft-tritopological spaces.

\section{PRELIMINARIES}

In the following, some foundations concepts about softsets, soft-topological spaces and soft-tritopological spaces are given.

Definition 2.1[1] Let the set $X$ be an initial universe and $\mathbb{E}$ be a set of parameters. Let $\mathcal{P}(\mathrm{X})$ denotes the power set of $\mathrm{X}$ and $\mathrm{A}$ be a non-empty subset of $\mathbb{E}$. A pair $\left(F_{v} A\right)$ is said to be a soft-set over $\mathrm{X}$, where $\mathrm{F}$ is a mapping given by Fi $\mathbb{A} \rightarrow \mathscr{P}(\mathrm{X})$.In other words, a soft-set over $\mathrm{X}$ is a parametrized family of subsets of the universe $X$. For $e \in A$, $F(e)$ may be considered as the set of $\mathrm{e}$-approximate elements of the soft-set $(F, A)$. Clear that, a soft-set is not a set.

Definition 2.2 [3] The complement (relative complement) of a soft-set $\left(F_{v} \mathbb{E}\right)$ is denoted by $\left(F_{v} \mathbb{E}\right)^{c}$ and is defined by $\left(F_{v} \mathbb{E}\right)^{c}=\left(\mathcal{F}^{c}{ }_{s} \mathbb{E}\right)$ where $\mathcal{F}^{c}{ }_{\mathrm{x}} \mathbb{E} \rightarrow \mathcal{P}(\mathrm{X})$ is a mapping given by $\mathcal{F}^{C}(\mathrm{e})=\mathrm{X}-\mathrm{F}(\mathrm{e})$ for all $\mathrm{e} \in \mathbb{E}$.

Definition 2.3 [3] Let $Y$ be a non-empty subset of $X$, then $Y$ denotes the soft-set $\left(\mathrm{Y}_{x} \mathbb{E}\right)$ over $\mathrm{X}$ for which $\mathrm{Y}(\mathrm{e})=\mathrm{Y}$, for all $\mathrm{e} \in \mathbb{E}$. In particular, $\left(\mathrm{X}_{v} \mathbb{E}\right)$ will be denoted by $X$. 
Definition 2.4 [3] Let $x \in \mathrm{X}$. Then $\left(x_{r} \mathbb{E}\right)$ denotes the sof $t$-set over $\mathrm{X}$ for which $x(e)=\{x\}$, for all $\mathrm{e} \in \mathbb{E}$.

Definition 2.5 [3] Let $\left(F_{v} \mathbb{E}\right)$ be a soft -set over $\mathrm{X}$ and $x \in \mathrm{X}$. We say that $x \in\left(F_{0} \mathbb{E}\right)$ read as $x$ belongs to the soft-set $\left(F_{s} \mathbb{E}\right)$ whenever $x \in \mathbb{F}(e)$ for all $e \in \mathbb{E}$. Note that for any $x \in \mathrm{X}, x \notin\left(F_{v} \mathbb{E}\right)$, if $x \in \mathbb{F}(e)$ for some $e \in \mathbb{E}$.

Definition 2.6 [3] The Union of two soft-sets $\left(F_{v} \mathbb{E}\right)$ and $\left(G_{s} \mathbb{E}\right)$ over the common universe $\mathrm{X}$ is the soft-set $\left(\mathrm{H}_{s} \mathbb{E}\right)$ where $H(e)=F(e) \cup G(e)$ for all $e \in \mathbb{E}$. We write $\left(F_{v} \mathbb{E}\right) \cup(G, \mathbb{E})=\left(H_{s}, \mathbb{E}\right)$.

Definition 2.7 [3] The soft-intersection of two soft-sets $(F, \mathbb{E})$ and $(G, \mathbb{E})$ over the common universe $\mathrm{X}$ is the soft-set $\left(H_{s} \mathbb{E}\right)$ where $\mathrm{H}(e)=\mathrm{F}(e) \cap \mathrm{G}(e)$ for all $\theta \in \mathbb{E}$. We write $\left(F_{v} \mathbb{E}\right) \cap\left(G_{v} \mathbb{E}\right)=\left(\mathrm{H}_{v} \mathbb{E}\right)$.

Definition 2.8 [4] Let $\tau$ be the collection of soft-sets over X, then $\tau$ is said to be a soft-topology on $\mathrm{X}$, if

(1) the soft-sets $\Phi, X$ belongs to $\pi$.

(2) the union of any number of soft $t$-sets in $\tau$ belongs to $\tau$.

(3) the intersection of any two soft - sets in $\tau$ belongs to $\tau$.

Then, the triple $\left(x_{v} \tau_{v} \mathbb{E}\right)$ is called a soft-topological space over $\mathrm{X}$.

Definition 2.9 [4] Let $\left(X_{s} \tau_{s} \mathbb{E}\right)$ be a soft-topological space over $\mathrm{X}$, then the members of $\tau$ are said to be soft-open sets in $X$.

Definition 2.10 [4] Let $\left(x_{v} \tau_{s} \mathbb{E}\right)$ be a soft-topological space over X. A soft-open set $\left(F_{0} \mathbb{E}\right)$ over $\mathrm{X}$ is said to be a soft-closed set in $\mathrm{X}$, if its complement $\left(\mathrm{F}_{v} \mathbb{E}\right)^{t}$ belongs to $\tau$.

Definition 2.11 [7] Let $\left(X_{v} \tau_{1}, \mathbb{E}\right),\left(X_{v} \tau_{2}, \mathbb{E}\right)$ and $\left(x_{v} \tau_{3}, \mathbb{E}\right)$ be the three soft-topological spaces on $x$. Then $\left(x_{v}, \tau_{1}, \tau_{2}, \tau_{7}, \mathbb{E}\right)$ is called a soft-tritopological space. The three soft-topological spaces $\left(X_{v} \tau_{1}, \mathbb{E}\right),\left(X_{v} \tau_{2}, \mathbb{E}\right)$ and $\left(X_{v}, \tau_{7}, \mathbb{E}\right)$ are independently satisfy the axioms of soft-topological space.

Definition 2.12 [7] Let $\left(x_{v} \tau_{1}, \tau_{2}, \tau_{\mathrm{g}}, \mathbb{E}\right)$ be a soft-tritopological space, then a soft-set $\left(F_{v} \mathbb{E}\right)$ in the universe $X$ is called a soft $-\delta^{*}-$ open set iff:

$\left(F_{v} \mathbb{E}\right) \subseteq S_{*} \tau_{1}-\operatorname{int}\left(S_{*} \tau_{2}-\operatorname{cl}\left(S_{*} \tau_{a}-\operatorname{int}\left(F_{v} \mathbb{E}\right)\right)\right)$. The complement of soft- $\delta^{*}$-open set is called a soft $-\delta^{*}-$ closed set. The family of all soft $-\delta^{*}-$ open sets is denoted by $5 . \delta^{*} \mathrm{O}(X)$. And the family of all soft $-\delta^{*}-$ closed sets is denoted by $\mathrm{S}_{*} \delta^{*} \mathrm{C}(X)$.

Definition 2.13 [15] A soft-set $\left(F_{0} \mathbb{E}\right)$ in a soft-topological space $\left(X_{v} \tau_{s} \mathbb{E}\right)$, then:

(i) The soft- $\delta^{*}$-closure of $\left(F_{x} \mathbb{E}\right)$, denoted by $S . \delta^{*}-\operatorname{cl}\left(F_{v} \mathbb{E}\right)$ is defined by:

$S . \delta^{*}-c l(F, \mathbb{E})=\bigcap\left\{(G, \mathbb{E}) s\left(F_{v} \mathbb{E}\right) \subseteq\left(G_{v} \mathbb{E}\right)\right.$, and $(G, \mathbb{E})$

is soft $-\delta^{*}-$ closed $\}$ (ii) The soft $-\delta^{*}$-interior of $\left(F_{v} \mathbb{E}\right)$, denoted by $S_{x} \hat{\delta}^{*}-\operatorname{int}\left(\mathrm{F}_{v} \mathbb{E}\right)$ is defined by:

$S . \delta^{*}-\operatorname{int}\left(F_{v} \mathbb{E}\right)=\cup\left\{\left(H_{v} \mathbb{E}\right):\left(H_{v} \mathbb{E}\right) \subseteq\left(F_{v} \mathbb{E}\right), \operatorname{and}\left(H_{s} \mathbb{E}\right) \quad\right.$ is soft $-\delta^{*}-$ open $\}$

Definition 2.14 [16] Let $\left(x_{v} \tau_{1}, \tau_{2}, \tau_{3,}, \mathbb{E}\right)$ be a soft-tritopological space over $\mathrm{X}, \mathrm{Y} \neq \emptyset$ and $\mathrm{Y} \subseteq \mathrm{X}$. Then the soft-relative space (soft-subspace) for $\left(X_{v} \tau_{10}, \tau_{2}, \tau_{y}, \mathbb{E}\right)$ on $Y$ (with respect to a soft $-\delta^{*}$-open set) is the collection $S \cdot \delta^{*} . O(x)_{\mathrm{Y}}$, given by:

$S_{x} \delta^{*}, O(X)_{\mathrm{Y}}=\left\{\left(F_{F^{*}} \mathbb{E}\right)=y \cap\left(F_{x} \mathbb{E}\right) \|\left(F_{x} \mathbb{E}\right) \in S_{x} \delta^{*} \cdot O(X)\right\}$, where $y$ is the soft $-\operatorname{set}\left(\mathrm{Y}_{v} \mathbb{E}\right)$ and $F_{\mathrm{Y}}(e)=\mathrm{Y} \cap \mathrm{F}(\mathrm{e})$, for all $\theta \in \mathbb{E}$. The members of $S . \delta^{*}, O(X)_{Y}$ are said to be soft $-\delta_{\mathrm{Y}}^{*}$-open sets in $Y$.

Note 2.15 Throughout this paper, $x$ [for simply] denote the soft-tritopological space over $\mathrm{X}$, [and simply $\mathbb{Y}$ ] denote to the soft-subspace of a soft-tritopological space $X$.

\section{Soft $-\delta^{8}-\mathrm{T}_{\mathrm{i}} ;(\mathrm{i}=0,1,2)$ spaces}

In this section the definition and some foundations of separation axioms in soft-tritopological spaces (soft $-\delta^{*}-\mathrm{T}_{0}$, soft $-\delta^{*}-\mathrm{T}_{1}$, soft $-\delta^{*}-\mathrm{T}_{2}$ ) w.r.t. ordinary points are given.

Definition 3.1. Let $\left(x_{v}, \tau_{1}, \tau_{2}, \tau_{2}, \mathbb{E}\right)$ be a soft-tritopological space over $\mathrm{X}$, and $x_{1}, x_{2} \in \mathrm{X}$ such that $x_{1} \neq x_{2}$. If there exist soft- $\delta^{*}$-open sets $\left(F_{1,} \mathbb{E}\right)$ and $\left(F_{2,} \mathbb{E}\right)$ such that " $x_{1} \in\left(F_{1}, \mathbb{E}\right), \quad x_{2} \notin\left(F_{1}, \mathbb{E}\right)$ " or “ $x_{1} \notin\left(F_{2}, \mathbb{E}\right)$, $x_{2} \in\left(F_{2}, \mathbb{E}\right)$ ", then $\left(x_{v}, \tau_{1}, \tau_{2}, \tau_{2}, \mathbb{E}\right)$ is said to be a soft $-\delta^{*}-\mathrm{T}_{0}-$ space.

Example 3.2. Let $\mathrm{X}=\left\{x_{1}, x_{2}\right\}$ be the universe set and $\mathbb{E}=\left\{e_{1}, e_{2}\right\}$ be the set of parameters, Then $\left(\mathrm{X}_{v} \mathbb{E}\right)=\left\{\left(e_{1},\left\{x_{1}, x_{2}\right\}\right),\left(e_{2},\left\{x_{1}, x_{2}\right\}\right)\right\}$. By [5], its cardinality is defined by $|\mathcal{P}(X)|=2^{\sum_{\varepsilon=\mathrm{a}}|\mathcal{F}(\mathrm{e})|}$, where $|\mathcal{F}(\mathrm{e})|$ is the cardinality of $\mathcal{F}(\mathrm{e})$. (i.e. $|\mathcal{P}(X)|=2^{4}=16$ soft-set).

Let $\left(X_{v}, \tau_{1}, \mathbb{E}\right), \quad\left(X_{v}, \tau_{2}, \mathbb{E}\right)$ and $\left(X_{v}, \tau_{7}, \mathbb{E}\right)$ be the three soft-topological spaces on $\mathrm{X}$, where their topologies defined as follows:

$$
\begin{aligned}
& \tau_{1}=\left\{\Phi, X_{v}\left(\mathcal{M}_{1}, \mathbb{E}\right),\left(\mathcal{M}_{2}, \mathbb{E}\right)\right\}, \\
& \tau_{2}=\left\{\Phi_{v} x_{v}\left(\mathrm{G}_{1,} \mathbb{E}\right),\left(\mathrm{G}_{2}, \mathbb{E}\right)\right\} \text { and } \\
& M_{1}\left(e_{1}\right)=\emptyset \quad, \quad M_{1}\left(e_{2}\right)=\emptyset \\
& M_{2}\left(e_{1}\right)=\mathrm{X} \quad, \quad M_{2}\left(e_{2}\right)=\mathrm{X} \\
& \mathrm{G}_{1}\left(e_{1}\right)=\mathrm{X} \quad, \quad \mathrm{G}_{1}\left(e_{2}\right)=\left\{x_{1}\right\} \\
& \mathrm{G}_{2}\left(e_{1}\right)=\emptyset \quad, \quad \mathrm{G}_{2}\left(e_{2}\right)=\left\{x_{1}\right\} \\
& \mathscr{H}\left(e_{1}\right)=\mathrm{X} \quad, \quad \mathscr{H}\left(e_{2}\right)=\left\{x_{1}\right\}
\end{aligned}
$$$$
\tau_{\mathrm{a}}=\left\{\Phi_{x} x_{\mathrm{v}}\left(\mathcal{H}_{1,} \mathbb{E}\right)\right\} \quad \text {. Where }\left(\mathcal{M}_{1 \mathrm{v}} \mathbb{E}\right),\left(\mathcal{M}_{2}, \mathbb{E}\right) \text {, }
$$$$
\left(\mathrm{G}_{1}, \mathbb{E}\right),\left(\mathrm{G}_{2}, \mathbb{E}\right),\left(\mathcal{H}_{1}, \mathbb{E}\right) \text { are soft-open sets defined as : }
$$

The complement of the soft-open sets of $\tau_{2}$ Are ;

$\mathrm{G}_{1}^{\mathrm{C}}\left(e_{1}\right)=\emptyset \quad, \quad \mathrm{G}_{1}^{\mathrm{C}}\left(e_{2}\right)=\left\{x_{2}\right\}$ 
$\mathrm{G}_{2}{ }^{\mathrm{C}}\left(e_{1}\right)=\mathrm{X} \quad, \quad \mathrm{G}_{2}{ }^{\mathrm{C}}\left(e_{2}\right)=\left\{x_{2}\right\}$

Hence the family of all soft- $\delta^{*}$-open sets of the soft-tritopological space $\left(x_{v}, \tau_{1}, \tau_{2}, \tau_{3}, \mathbb{E}\right)$ is,

S. $\delta^{*} \mathrm{O}(X)=\left\{X_{v} \Phi_{v}\left(\mathcal{F}_{v} \mathbb{E}\right)\right\}$.

$\mathcal{F}\left(e_{1}\right)=\mathrm{X} \quad, \quad \mathcal{F}\left(e_{2}\right)=\left\{x_{1}\right\}$

Let $x_{1}, x_{2} \in \mathrm{X}$ such that $x_{1} \neq x_{2}$

$\exists\left(\mathcal{F}_{x} \mathbb{E}\right) \in \mathrm{S}_{x} \delta^{*}, \mathrm{O}(X)$ such that

$x_{1} \in\left(\mathcal{F}_{x} \mathbb{E}\right){ }_{o} x_{2} \notin\left(\mathcal{F}_{x} \mathbb{E}\right)$

Thus $\left(X_{v}, \tau_{1}, \tau_{2}, \tau_{2}, \mathbb{E}\right)$ is a soft $-\delta^{*}-\mathrm{T}_{0}-$ space.

Proposition 3.3. Let $\left(x_{v} \tau_{1}, \tau_{2}, \tau_{7 x}, \mathbb{E}\right)$ be a softtritopological space over $\mathrm{X}$ and $\mathrm{Y}$ be a non-empty subset of $\mathrm{X}$. If $X$ is a soft $-\delta^{*}-\mathrm{T}_{0}-$ space then the soft-subspace $\mathrm{Y}$ is a soft $-\delta^{*}-\mathrm{T}_{0}-$ space.

Proof. Let $Y_{1} \neq y_{2} \in Y$. There exists a two soft $-\delta^{*}$-open sets $\left(\mathcal{A}_{s} \mathbb{E}\right)_{v}\left(\mathcal{B}_{v} \mathbb{E}\right)$ in $X$ (in $\mathrm{S}_{s} \delta^{*} \mathrm{O}(X)$ ), such that $Y_{1} \in\left(\mathcal{A}_{v} \mathbb{E}\right), \quad \mathrm{Y}_{2} \notin(\mathcal{A}, \mathbb{E})$ or $\mathrm{Y}_{1} \notin\left(\mathcal{B}_{v} \mathbb{E}\right), \mathrm{Y}_{2} \in\left(\mathcal{B}_{v} \mathbb{E}\right)$. Now, $Y_{1} \in Y$ implies that $Y_{1} \in\left(Y_{v} \mathbb{E}\right)$. So $Y_{1} \in\left(Y_{v} \mathbb{E}\right)$ and $Y_{1} \in\left(\mathcal{A}_{s} \mathbb{E}\right)$. Hence $Y_{1} \in\left(\mathrm{Y}_{v} \mathbb{E}\right) \cap\left(\mathcal{A}_{s} \mathbb{E}\right)=\left(\mathcal{A}_{\mathrm{Y}} \mathbb{E}\right)$ where $\left(\mathcal{A}_{s} \mathbb{E}\right) \in \mathrm{S} . \delta^{*} \mathrm{O}(X)$. Consider $y_{2} \notin(\mathcal{A}, \mathbb{E})$, this means that $Y_{2} \notin \mathcal{A}(\mathrm{e})$ for some $\mathrm{e} \in \mathbb{E}$. Then $\mathrm{y}_{2} \notin \mathrm{Y} \cap \mathcal{A}(\mathrm{e})$ $=\mathrm{Y}(\mathrm{e}) \cap \mathcal{A}(\mathrm{e})$. Therefore $\mathrm{Y}_{2} \mathbb{E}\left(\mathrm{Y}_{v} \mathbb{E}\right) \cap\left(\mathcal{A}_{v} \mathbb{E}\right)=\left(\mathcal{A}_{\mathrm{Y}} \mathbb{E}\right)$. Similarly it can be proved that If $Y_{2} \in(\mathcal{B}, \mathbb{E})$ and $Y_{1} \notin(\mathcal{B}, \mathbb{E})$ then $Y_{2} \in\left(\mathcal{B}_{\mathrm{Y}} \mathbb{E}\right), Y_{1} \notin\left(\mathcal{B}_{\mathrm{Y}}, \mathbb{E}\right)$.

Thus the soft-subspace $\mathbb{Y}$ is a soft $-\delta^{*}-\mathrm{T}_{0}-$ space.

Definition 3.4. Let $\left(X_{v}, \tau_{1}, \tau_{2}, \tau_{7}, \mathbb{E}\right)$ be a soft-tritopological space over $\mathrm{X}$, and $x_{1}, x_{2} \in \mathrm{X}$ such that $x_{1} \neq x_{2}$. If there exist soft- $\delta^{*}$-open sets $\left(F_{1}, \mathbb{E}\right)$ and $\left(F_{2}, \mathbb{E}\right)$ such that “ $x_{1} \in\left(F_{1}, \mathbb{E}\right), \quad x_{2} \notin\left(F_{1}, \mathbb{E}\right)$ " and “ $x_{1} \notin\left(F_{2}, \mathbb{E}\right)$, $x_{2} \in\left(F_{2}, \mathbb{E}\right)$ ", then $\left(x_{v}, \tau_{1}, \tau_{2}, \tau_{2,}, \mathbb{E}\right)$ is said to be a soft $-\delta^{*}-\mathrm{T}_{1}-$ space.

Example 3.5. Let $\mathrm{X}=\left\{x_{1}, x_{2}\right\}$ be the universe set and $\mathbb{E}=\left\{e_{1}, \epsilon_{2}\right\}$ be the set of parameters, Then $\left(\mathrm{X}_{v} \mathbb{E}\right)=\left\{\left(e_{1 v}\left\{x_{1}, x_{2}\right\}\right),\left(e_{2 v},\left\{x_{1}, x_{2}\right\}\right)\right\}$. By [5], its cardinality is defined by $|\mathcal{P}(X)|=2^{\sum \boldsymbol{V}_{\mathrm{E}}|\mathcal{F}(\mathrm{e})|}$, where $|\mathcal{F}(\mathrm{e})|$ is the cardinality of $\mathcal{F}\left(\right.$ e). ( i.e. $|\mathcal{P}(X)|=2^{4}=16$ soft-set).

Let $\left(x_{v}, \tau_{1}, \mathbb{E}\right), \quad\left(x_{v}, \tau_{2}, \mathbb{E}\right)$ and $\left(x_{v}, \tau_{2}, \mathbb{E}\right)$ be the three soft-topological spaces on $\mathrm{X}$, where their soft-topologies defined as follows:

$$
\begin{aligned}
& \tau_{1}=\left\{\Phi_{v} x_{v}\left(M_{1}, \mathbb{E}\right),\left(M_{2}, \mathbb{E}\right)\right\}, \\
& \tau_{2}=\left\{\Phi_{v}, x_{v}\left(\mathrm{G}_{1}, \mathbb{E}\right),\left(\mathrm{G}_{2}, \mathbb{E}\right),\left(\mathrm{G}_{\mathrm{g}}, \mathbb{E}\right),\left(\mathrm{G}_{4}, \mathbb{E}\right)\right\} \text { and } \\
& \tau_{a}=\left\{\Phi_{s} x_{v}\left(\mathscr{H}_{1}, \mathbb{E}\right),\left(\mathscr{H}_{2}, \mathbb{E}\right),\left(\mathscr{H}_{a}, \mathbb{E}\right),\left(\mathscr{H}_{4}, \mathbb{E}\right)\right\} \text { Where } \\
& \left(\mathcal{M}_{1}, \mathbb{E}\right),\left(\mathcal{M}_{2}, \mathbb{E}\right),\left(\mathrm{G}_{1}, \mathbb{E}\right),\left(\mathrm{G}_{2}, \mathbb{E}\right),\left(\mathrm{G}_{2}, \mathbb{E}\right),\left(\mathrm{G}_{4}, \mathbb{E}\right), \quad\left(\mathcal{H}_{1}, \mathbb{E}\right), \\
& \left(\mathcal{H}_{2}, \mathbb{E}\right)_{x}\left(\mathcal{H}_{2 x} \mathbb{E}\right) \text { and }\left(\mathcal{H}_{4}, \mathbb{E}\right) \text { are soft - open sets defined as: } \\
& M_{1}\left(e_{1}\right)=\left\{x_{1}\right\} \\
& M_{2}\left(e_{1}\right)=\left\{x_{1}\right\} \quad, \quad M_{2}\left(e_{2}\right)=\left\{x_{2}\right\} \\
& \mathrm{G}_{1}\left(e_{1}\right)=\mathrm{X} \quad, \quad \mathrm{G}_{1}\left(e_{2}\right)=\emptyset \\
& \mathrm{G}_{2}\left(e_{1}\right)=\left\{x_{2}\right\} \quad, \quad \mathrm{G}_{2}\left(e_{2}\right)=\left\{x_{1}\right\}
\end{aligned}
$$

$$
\begin{aligned}
& \mathrm{G}_{\mathrm{a}}\left(e_{1}\right)=\left\{x_{2}\right\} \quad, \quad \mathrm{G}_{\mathrm{a}}\left(e_{2}\right)=\emptyset \\
& \mathrm{G}_{4}\left(e_{1}\right)=\mathrm{X} \quad, \quad \mathrm{G}_{4}\left(e_{2}\right)=\left\{x_{1}\right\} \\
& \mathscr{H}_{1}\left(e_{1}\right)=\mathrm{X} \quad, \quad \mathscr{H}_{1}\left(e_{2}\right)=\left\{x_{2}\right\} \\
& \mathscr{H}_{2}\left(e_{1}\right)=\left\{x_{1}\right\} \quad, \quad \mathscr{H}_{2}\left(e_{2}\right)=\mathrm{X} \\
& \mathscr{H}_{a}\left(e_{1}\right)=\left\{x_{2}\right\} \quad, \quad \mathscr{H}_{a}\left(e_{2}\right)=\emptyset \\
& \mathscr{H}_{4}\left(e_{1}\right)=\left\{x_{1}\right\} \quad, \quad \mathscr{H}_{4}\left(e_{2}\right)=\left\{x_{2}\right\}
\end{aligned}
$$

The complement of the soft-open sets of soft-topology $\tau_{2}$ Are ;

$$
\begin{array}{lll}
\mathrm{G}_{1}{ }^{\mathrm{C}}\left(e_{1}\right)=\emptyset & , & \mathrm{G}_{1}{ }^{\mathrm{C}}\left(e_{2}\right)=\mathrm{X} \\
\mathrm{G}_{2}{ }^{\mathrm{C}}\left(e_{1}\right)=\left\{x_{1}\right\} & , & \mathrm{G}_{2}{ }^{\mathrm{C}}\left(e_{2}\right)=\left\{x_{2}\right\} \\
\mathrm{G}_{\mathrm{g}}{ }^{\mathrm{C}}\left(e_{1}\right)=\left\{x_{1}\right\} & , & \mathrm{G}_{9}{ }^{\mathrm{C}}\left(e_{2}\right)=\mathrm{X} \\
\mathrm{G}_{4}{ }^{\mathrm{C}}\left(e_{1}\right)=\emptyset & , & \mathrm{G}_{4}{ }^{\mathrm{C}}\left(e_{2}\right)=\left\{x_{2}\right\}
\end{array}
$$

Hence the family of all soft- $\delta^{*}$-open sets of the soft-tritopological space $\left(X_{v}, \tau_{1}, \tau_{2}, \tau_{3}, \mathbb{E}\right) \quad$ is, $\mathrm{S}_{\mathrm{s}} \delta^{*}, \mathrm{O}(X)=\left\{X_{v} \Phi_{s}\left(\mathcal{F}_{1,}, \mathbb{E}\right),\left(\mathcal{F}_{2}, \mathbb{E}\right),\left(\mathcal{F}_{2 x}, \mathbb{E}\right)\right\}$.

$$
\begin{array}{lll}
\mathcal{F}_{1}\left(e_{1}\right)=\left\{x_{1}\right\} & , & \mathcal{F}_{1}\left(e_{2}\right)=\mathrm{X} \\
\mathcal{F}_{2}\left(e_{1}\right)=\mathrm{X} & , & \mathcal{F}_{2}\left(e_{2}\right)=\left\{x_{2}\right\} \\
\mathcal{F}_{2}\left(e_{1}\right)=\left\{x_{1}\right\} & , & \mathcal{F}_{2}\left(e_{2}\right)=\left\{x_{2}\right\}
\end{array}
$$

Let $x_{1} x_{2} \in \mathrm{X}_{x}$ such that $x_{1} \neq x_{2}$

$\exists\left(\mathcal{F}_{1 x} \mathbb{E}\right),\left(\mathcal{F}_{2}, \mathbb{E}\right) \in \mathrm{S}, \delta^{*}, \mathrm{O}(X)$ such that

$$
\begin{aligned}
& x_{1} \in\left(\mathcal{F}_{1,}, \mathbb{E}\right), x_{2} \notin\left(\mathcal{F}_{1}, \mathbb{E}\right) \text { and } \\
& x_{2} \in\left(\mathcal{F}_{2}, \mathbb{E}\right), x_{1} \notin\left(\mathcal{F}_{2}, \mathbb{E}\right)
\end{aligned}
$$

Thus $\left(X_{v} \tau_{1}, \tau_{2}, \tau_{7}, \mathbb{E}\right)$ is a soft $-\delta^{*}-\mathrm{T}_{1}-$ space.

Remark 3.6. If $\left(X_{v}, \tau_{1}, \tau_{2}, \tau_{3}, \mathbb{E}\right)$ is a soft $-\delta^{*}-T_{1}-$ space then $\left(\mathrm{X}, \delta^{*} \cdot \tau_{\epsilon}\right)$ may not be a $\mathrm{T}_{1}$-space for every parameter $\mathrm{e} \in \mathbb{E}$. Where $\delta^{*} \cdot \tau_{\mathrm{e}}=\left\{\mathrm{F}(\mathrm{e}) \mid\left(\mathrm{F}_{v} \mathbb{E}\right) \in \mathrm{S}_{*} \delta^{*} \mathrm{o} \mathrm{O}(X)\right\}$ [15].

Example 3.7. Clearly in example 3.5 above, Neither

$\left(\mathrm{X}, \delta^{*} \cdot \tau_{\varepsilon_{1}}\right)$ nor $\left(\mathrm{X}, \delta^{*} \cdot \tau_{s_{2}}\right)$ is a $\mathrm{T}_{1}$-space. Where $\delta^{*} \cdot \tau_{s_{1}}=\left\{\mathrm{X}_{0} \emptyset,\left\{x_{1}\right\}\right\}$ and $\delta^{*} \cdot \tau_{s_{2}}=\left\{\mathrm{X}_{0} \emptyset,\left\{x_{2}\right\}\right\}$

Proposition 3.8. Every soft $-\delta^{*}-\mathrm{T}_{1}-$ space is a sof $t-\delta^{*}-T_{0}-$ space.

Proof. Let $\left(x_{v}, \tau_{10}, \tau_{2}, \tau_{2}, \mathbb{E}\right)$ be a soft-tritopological space over $\mathrm{X}$ and $x_{1}, x_{2} \in \mathrm{X}$ such that $x_{1} \neq x_{2}$. If $\left(x_{v}, \tau_{1}, \tau_{2}, \tau_{2}, \mathbb{E}\right)$ is a soft $-\delta^{*}-\mathrm{T}_{1}$-space then there exist soft $-\delta^{*}$-open sets $\left(\mathcal{A}_{v} \mathbb{E}\right)$ and $\left(\mathcal{B}_{v} \mathbb{E}\right)$ such that $x_{1} \in\left(\mathcal{A}_{v} \mathbb{E}\right), x_{2} \notin\left(\mathcal{A}_{s} \mathbb{E}\right)$

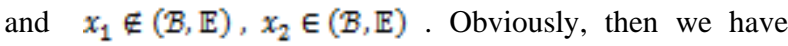

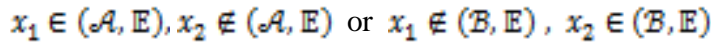

Thus $\left(X_{v}, \tau_{1}, \tau_{2}, \tau_{y}, \mathbb{E}\right)$ is a soft $-\delta^{*}-\mathrm{T}_{0}-$ space.

Proposition 3.9. Let $\left(x_{v} \tau_{1}, \tau_{2}, \tau_{7,}, \mathbb{E}\right)$ be a softtritopological space over $\mathrm{X}$ and $\mathrm{Y}$ be a non-empty subset of $\mathrm{X}$. If $X$ is a soft $-\delta^{*}-\mathrm{T}_{1}$-space then the soft-subspace $\mathbb{Y}$ is a soft $-\delta^{*}-\mathrm{T}_{1}-$ space.

Proof. Let $y_{1} \neq y_{2} \in Y$. There exists a two soft $-\delta^{*}-$ open 
sets $\left(\mathcal{A}_{s} \mathbb{E}\right),\left(B_{s} \mathbb{E}\right)$ in $X$ (in $\mathrm{S}_{x} \delta^{*}{ }^{*} \mathrm{O}(X)$ ), such that

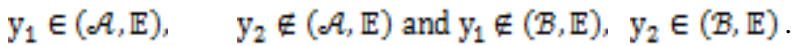
Now $y_{1} \in Y$ implies that $y_{1} \in\left(Y_{v} \mathbb{E}\right)$. So $y_{1} \in\left(Y_{v} \mathbb{E}\right)$ and $Y_{1} \in\left(\mathcal{A}_{s} \mathbb{E}\right)$.

Hence $\quad Y_{1} \in\left(\mathrm{Y}_{v} \mathbb{E}\right) \cap\left(\mathcal{A}_{s} \mathbb{E}\right)=\left(\mathcal{A}_{\mathrm{Y}} \mathbb{E}\right) \quad$ where $\left(\mathcal{A}_{s} \mathbb{E}\right) \in \mathrm{S} . \delta^{*}{ }_{x} \mathrm{O}(X)$. Consider $Y_{2} \notin\left(\mathcal{A}_{s} \mathbb{E}\right)$, this means that $Y_{2} \notin \mathcal{A}(\mathrm{e})$ for some $\mathrm{e} \in \mathbb{E}$. Then $y_{2} \notin \mathrm{Y} \cap \mathcal{A}(\mathrm{e})$ $=\mathrm{Y}(\mathrm{e}) \cap \mathcal{A}(\mathrm{e})$. Therefore $\mathrm{Y}_{2} \mathbb{E}\left(\mathrm{Y}_{x} \mathbb{E}\right) \cap\left(\mathcal{A}_{s} \mathbb{E}\right)=\left(\mathcal{A}_{\mathrm{Y}} \mathbb{E}\right)$. Similarly it can be proved that If $Y_{2} \in(\mathcal{B}, \mathbb{E})$ and $Y_{1} \notin(\mathcal{B}, \mathbb{E})$ then $Y_{2} \in\left(\mathcal{B}_{\mathrm{Y}}, \mathbb{E}\right)$ and $Y_{1} \notin\left(\mathcal{B}_{\mathrm{Y}} \mathbb{E}\right)$. Thus $\mathbb{Y}$ is a soft $-\delta^{*}-\mathrm{T}_{1}-$ space.

Definition 3.10. Let $\left(x_{v}, \tau_{1}, \tau_{2}, \tau_{2}, \mathbb{E}\right)$ be a softtritopological space over $\mathrm{X}$, and $x_{1}, x_{2} \in \mathrm{X}$ such that $x_{1} \neq x_{2}$. If there exist soft $-\delta^{*}-$ open sets $\left(F_{1}, \mathbb{E}\right)$ and $\left(F_{2}, \mathbb{E}\right)$ such that " $x_{1} \in\left(F_{1}, \mathbb{E}\right), x_{2} \in\left(F_{2}, \mathbb{E}\right)$ and $\left(F_{1}, \mathbb{E}\right) \cap\left(F_{2}, \mathbb{E}\right)=\Phi$ ", then $\left(X_{v}, \tau_{1}, \tau_{2}, \tau_{3}, \mathbb{E}\right)$ is said to be a soft $-\delta^{*}-\mathrm{I}_{2}-$ space.

Example 3.11. Let $\mathrm{X}=\left\{x_{1}, x_{2}\right\}$ be the universe set and $\mathbb{E}=\left\{e_{1}, e_{2}\right\}$ be the set of parameters, Then $\left(\mathrm{X}_{v} \mathbb{E}\right)=\left\{\left(e_{10},\left\{x_{1}, x_{2}\right\}\right),\left(e_{2},\left\{x_{1}, x_{2}\right\}\right)\right\}$. By [5], its cardinality is defined by $|\mathcal{P}(X)|=2^{\sum \in \mathrm{E}}|\mathcal{F}(\mathrm{e})|$, where $|\mathcal{F}(\mathrm{e})|$ is the cardinality of $\mathcal{F}\left(\right.$ e) . (i.e. $|\mathcal{P}(x)|=2^{4}=16$ soft-set).

Let $\left(X_{v}, \tau_{1}, \mathbb{E}\right), \quad\left(X_{v}, \tau_{2}, \mathbb{E}\right)$ and $\left(X_{v}, \tau_{g}, \mathbb{E}\right)$ be the three soft-topological spaces on $x$, and their soft-topologies defined as follows:

$\tau_{1}=\left\{\Phi_{v} x_{v}\left(M_{1}, \mathbb{E}\right),\left(M_{2}, \mathbb{E}\right),\left(M_{2}, \mathbb{E}\right),\left(M_{4}, \mathbb{E}\right)\right\}$,

$\tau_{2}=\left\{\Phi_{v} x_{v}\left(G_{1,} \mathbb{E}\right){ }_{v a s}\left(G_{7 v} \mathbb{E}\right)\right\}$ and

$\tau_{a}=\left\{\Phi_{v} X_{v}\left(\mathscr{H}_{1}, \mathbb{E}\right)_{v \infty n}\left(\mathcal{H}_{\mathcal{F}_{v}} \mathbb{E}\right)\right\}$. Where the soft-open sets over $\mathrm{X}$ of three soft -topologies, defined as follows:

\begin{tabular}{|c|c|c|}
\hline$M_{1}\left(e_{1}\right)=\left\{x_{1}\right\}$ & & $M_{1}\left(e_{2}\right)=\left\{x_{1}\right\}$ \\
\hline$M_{2}\left(e_{1}\right)=\left\{x_{2}\right\}$ & , & $M_{2}\left(e_{2}\right)=\left\{x_{2}\right\}$ \\
\hline$M_{a}\left(e_{1}\right)=\mathrm{X}$ & , & $M_{a}\left(\theta_{2}\right)=\left\{x_{1}\right\}$ \\
\hline$M_{4}\left(e_{1}\right)=\left\{x_{2}\right\}$ & , & $M_{4}\left(e_{2}\right)=\emptyset$ \\
\hline $\mathrm{G}_{1}\left(e_{1}\right)=\mathrm{X}$ & , & $\mathrm{G}_{1}\left(e_{2}\right)=\emptyset$ \\
\hline $\mathrm{G}_{2}\left(e_{1}\right)=\left\{x_{2}\right\}$ & , & $\mathrm{G}_{2}\left(e_{2}\right)=\left\{x_{2}\right\}$ \\
\hline $\mathrm{G}_{a}\left(e_{1}\right)=\left\{x_{1}\right\}$ & , & $\mathrm{G}_{a}\left(e_{2}\right)=\left\{x_{1}\right\}$ \\
\hline $\mathrm{G}_{4}\left(e_{1}\right)=\left\{x_{2}\right\}$ & , & $\mathrm{G}_{4}\left(e_{2}\right)=\emptyset$ \\
\hline $\mathrm{G}_{5}\left(e_{1}\right)=\left\{x_{1}\right\}$ & , & $\mathrm{G}_{5}\left(e_{2}\right)=\emptyset$ \\
\hline $\mathrm{G}_{6}\left(e_{1}\right)=\mathrm{X}$ & , & $\mathrm{G}_{6}\left(e_{2}\right)=\left\{x_{1}\right\}$ \\
\hline $\mathrm{G}_{7}\left(e_{1}\right)=\mathrm{X}$ & , & $\mathrm{G}_{7}\left(e_{2}\right)=\left\{x_{2}\right\}$ \\
\hline $\mathscr{H}_{1}\left(e_{1}\right)=\left\{x_{1}\right\}$ & , & $\mathscr{H}_{1}\left(e_{2}\right)=\left\{x_{1}\right\}$ \\
\hline $\mathscr{H}_{2}\left(e_{1}\right)=\left\{x_{2}\right\}$ & , & $\mathscr{H}_{2}\left(e_{2}\right)=\left\{x_{2}\right\}$ \\
\hline $\mathscr{H}_{\mathrm{a}}\left(e_{1}\right)=\mathrm{X}$ & 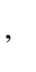 & $\mathscr{H}_{a}\left(e_{2}\right)=\emptyset$ \\
\hline $\mathscr{H}_{4}\left(e_{1}\right)=\left\{x_{2}\right\}$ & , & $\mathscr{H}_{4}\left(\epsilon_{2}\right)=\emptyset$ \\
\hline $\mathscr{H}_{5}\left(e_{1}\right)=\left\{x_{1}\right\}$ & , & $\mathscr{H}_{5}\left(e_{2}\right)=\emptyset$ \\
\hline
\end{tabular}

$\begin{array}{lll}\mathscr{H}_{6}\left(e_{1}\right)=\mathrm{X} & , & \mathscr{H}_{6}\left(e_{2}\right)=\left\{x_{1}\right\} \\ \mathscr{H}_{7}\left(e_{1}\right)=\mathrm{X} & , & \mathscr{H}_{7}\left(e_{2}\right)=\left\{x_{2}\right\}\end{array}$

The complement of the soft-open sets in $\tau_{2}$ Are ;

$\begin{array}{lll}\mathrm{G}_{1}{ }^{\mathrm{C}}\left(e_{1}\right)=\emptyset & , & \mathrm{G}_{1}{ }^{\mathrm{C}}\left(e_{2}\right)=\mathrm{X} \\ \mathrm{G}_{2}{ }^{\mathrm{C}}\left(e_{1}\right)=\left\{x_{1}\right\} & , & \mathrm{G}_{2}{ }^{\mathrm{C}}\left(e_{2}\right)=\left\{x_{1}\right\} \\ \mathrm{G}_{2} \mathrm{C}\left(e_{1}\right)=\left\{x_{2}\right\} & , & \mathrm{G}_{2}{ }^{\mathrm{C}}\left(e_{2}\right)=\left\{x_{2}\right\} \\ \mathrm{G}_{4}{ }^{\mathrm{C}}\left(e_{1}\right)=\left\{x_{1}\right\} & , & \mathrm{G}_{4}{ }^{\mathrm{C}}\left(e_{2}\right)=\mathrm{X} \\ \mathrm{G}_{5} \mathrm{C}^{\mathrm{C}}\left(e_{1}\right)=\left\{x_{2}\right\} & , & \mathrm{G}_{5}{ }^{\mathrm{C}}\left(e_{2}\right)=\mathrm{X} \\ \mathrm{G}_{6} \mathrm{C}\left(e_{1}\right)=\emptyset & , & \mathrm{G}_{6}{ }^{\mathrm{C}}\left(e_{2}\right)=\left\{x_{2}\right\} \\ \mathrm{G}_{7} \mathrm{C}\left(e_{1}\right)=\emptyset & , & \mathrm{G}_{7} \mathrm{C}\left(e_{2}\right)=\left\{x_{1}\right\}\end{array}$

Hence the family of all soft- $\delta^{*}$-open sets of the soft-tritopological space $\left(X_{v} \tau_{1}, \tau_{2}, \tau_{3}, \mathbb{E}\right) \quad$ is, S. $\delta^{*} \mathrm{O}(X)=\left\{X_{v} \Phi_{v}\left(\mathcal{F}_{\mathrm{i}}, \mathbb{E}\right)\right\}\left(\right.$ with $\left.\mathrm{i}=1{ }_{x}{ }_{v} \ldots{ }_{x} 7\right)$ defined as $\mathcal{F}_{1}\left(e_{1}\right)=\left\{x_{1}\right\} \quad, \quad \mathcal{F}_{1}\left(e_{2}\right)=\left\{x_{1}\right\}$

$\mathcal{F}_{2}\left(e_{1}\right)=\left\{x_{1}\right\} \quad, \quad \mathcal{F}_{2}\left(e_{2}\right)=\emptyset$

$\mathcal{F}_{a}\left(e_{1}\right)=\left\{x_{2}\right\} \quad, \quad \mathcal{F}_{a}\left(e_{2}\right)=\left\{x_{2}\right\}$

$\mathcal{F}_{4}\left(e_{1}\right)=\left\{x_{2}\right\} \quad, \quad \mathcal{F}_{4}\left(e_{2}\right)=\emptyset$

$\mathcal{F}_{5}\left(e_{1}\right)=\mathrm{X} \quad, \quad \mathcal{F}_{5}\left(e_{2}\right)=\left\{x_{1}\right\}$

$\mathcal{F}_{6}\left(e_{1}\right)=\mathrm{X} \quad, \quad \mathcal{F}_{6}\left(e_{2}\right)=\left\{x_{2}\right\}$

$\mathcal{F}_{7}\left(e_{1}\right)=\mathrm{X} \quad, \quad \mathcal{F}_{7}\left(e_{2}\right)=\emptyset$

If we take $x_{1}, x_{2} \in \mathrm{X}$ and $x_{1} \neq x_{2}$

$\exists\left(\mathcal{F}_{1}, \mathbb{E}\right),\left(\mathcal{F}_{\mathrm{g},} \mathbb{E}\right) \in \mathrm{S}, \delta^{*}, \mathrm{O}(\mathcal{X})$ such that

$x_{1} \in\left(\mathcal{F}_{1,} \mathbb{E}\right)$ and $x_{2} \in\left(\mathcal{F}_{a x} \mathbb{E}\right)$,

$\mathcal{F}_{1}\left(e_{1}\right) \cap \mathcal{F}_{9}\left(e_{1}\right)=\emptyset$

$\mathcal{F}_{1}\left(e_{2}\right) \cap \mathcal{F}_{\mathrm{g}}\left(e_{2}\right)=\emptyset$

Then $\left(\mathcal{F}_{1}, \mathbb{E}\right) \cap\left(\mathcal{F}_{2}, \mathbb{E}\right)=\Phi$

Thus $\left(X_{v}, \tau_{1}, \tau_{2}, \tau_{2}, \mathbb{E}\right)$ is a soft $-\delta^{*}-\mathrm{T}_{2}-$ space.

Proposition 3.12. Let the soft-tritopological space $\left(X_{v} \tau_{1}, \tau_{2}, \tau_{2}, \mathbb{E}\right)$ is a soft $-\delta^{*}-\mathrm{T}_{2}-$ space over $\mathrm{X}$, then $\left(\mathrm{X}, \delta^{*} \cdot \tau_{e}\right)$ is a $\mathrm{T}_{2}-$ space for every parameter $\mathrm{e} \in \mathbb{E}$.

Proof. Suppose that $\left(x_{v}, \tau_{1}, \tau_{2}, \tau_{2,}, \mathbb{E}\right)$ is a soft $-\delta^{*}-\mathrm{T}_{2}-$ space over $\mathrm{X}$. For any e $\in \mathbb{E}$, and $\delta^{*} \cdot \tau_{\mathrm{e}}=\left\{\mathrm{F}(\mathrm{e}) \mid\left(\mathrm{F}_{\mathrm{v}} \mathbb{E}\right) \in \mathrm{S} . \delta^{*}{ }^{*} \mathrm{O}(X)\right\}[15]$. Let $x_{1}, x_{2} \in \mathrm{X}$, such that $x_{1} \neq x_{2}$, there is a soft $-\delta^{*}$-open sets $\left(F_{10} \mathbb{E}\right)$ and $\left(\mathbb{F}_{2 x} \mathbb{E}\right)$ such that $x_{1} \in\left(F_{10} \mathbb{E}\right), \quad x_{2} \in\left(F_{2 x} \mathbb{E}\right)$ and $\left(F_{1}, \mathbb{E}\right) \cap\left(F_{2}, \mathbb{E}\right)=\Phi$. This implies that $x_{1} \in F_{1}(e)$, $x_{2} \in F_{2}(\mathrm{e})$ and $F_{1}(\mathrm{e}) \cap F_{2}(\mathrm{e})=\emptyset$

Thus $\left(\mathrm{X}, \delta^{*} \cdot \tau_{\mathbb{E}}\right)$ is a $\mathrm{T}_{2}-$ space, for every parameter e $\in \mathbb{E}$.

Proposition 3.13. Every soft $-\delta^{*}-T_{2}-$ space is a sof $t-\delta^{*}-T_{1}-$ space.

Proof. Let $\left(X_{v} \tau_{1}, \tau_{2}, \tau_{2}, \mathbb{E}\right)$ be a soft-tritopological space over $\mathrm{X}$ and $x_{1 v}, x_{2} \in \mathrm{X}$ such that $x_{1} \neq x_{2}$. If $\left(X_{v} \tau_{1}, \tau_{2}, \tau_{3}, \mathbb{E}\right)$ is 
a soft $-\delta^{*}-T_{2}$-space then there exist two soft- $\delta^{*}$-open sets $\left(\mathcal{A}_{s} \mathbb{E}\right)$ and $\left(\mathcal{B}_{s} \mathbb{E}\right)$ such that $x_{1} \in\left(\mathcal{A}_{s} \mathbb{E}\right), x_{2} \in\left(\mathcal{B}_{s} \mathbb{E}\right)$ and $\left(\mathcal{A}_{s} \mathbb{E}\right) \cap\left(\mathcal{B}_{s} \mathbb{E}\right)=\Phi$.

Obviously, since $\left(\mathcal{A}_{v} \mathbb{E}\right) \cap\left(\mathcal{B}_{v} \mathbb{E}\right)=\Phi$, then we have $x_{1} \in\left(\mathcal{A}_{s} \mathbb{E}\right), x_{2} \notin\left(\mathcal{A}_{s} \mathbb{E}\right)$ and $x_{1} \notin\left(\mathcal{B}_{v} \mathbb{E}\right), x_{2} \in\left(\mathcal{B}_{v} \mathbb{E}\right)$. Thus $\left(X_{v}, \tau_{1}, \tau_{2}, \tau_{3}, \mathbb{E}\right)$ is a soft $-\delta^{*}-\mathrm{T}_{1}$-space.

Proposition 3.14. Let $\left(X_{v} \tau_{1}, \tau_{2}, \tau_{3}, \mathbb{E}\right)$ be a softtritopological space over $\mathrm{X}$ and $\mathrm{Y}$ be a non-empty subset of $\mathrm{X}$. If $X$ is a soft $-\delta^{*}-\mathrm{T}_{2}-$ space then the soft-subspace $Y$ is a soft $-\delta^{*}-\mathrm{T}_{2}-$ space.

Proof. Let $y_{1} \neq y_{2} \in Y$. There exists a two soft $-\delta^{*}-$ open sets $\left(\mathcal{A}_{s} \mathbb{E}\right),\left(\mathcal{B}_{s} \mathbb{E}\right)$ in $X$ (in $\mathrm{S}_{s} \delta^{*} \mathrm{O}(X)$ ), such that $Y_{1} \in\left(\mathcal{A}_{s} \mathbb{E}\right), Y_{2} \in\left(\mathcal{B}_{s} \mathbb{E}\right)$ and $\left(\mathcal{A}_{s} \mathbb{E}\right) \cap\left(\mathcal{B}_{v} \mathbb{E}\right)=\Phi$. So for each $\mathrm{e} \in \mathbb{E}, Y_{1} \in \mathcal{A}(\mathrm{e}), y_{2} \in \mathcal{B}(\mathrm{e})$ and $\mathcal{A}(\mathrm{e}) \cap B(\mathrm{e})=\emptyset$, this implies that $y_{1} \in \mathrm{Y} \cap \mathcal{A}(\mathrm{e}), \quad y_{2} \in \mathrm{Y} \cap B(\mathrm{e})$ and $\mathcal{A}(\mathrm{e}) \cap \mathcal{B}(\mathrm{e})=\emptyset$. Hence $Y_{1} \in\left(\mathcal{A}_{\mathrm{Y}} \mathbb{E}\right), Y_{2} \in\left(\mathcal{B}_{\mathrm{Y}} \mathbb{E}\right)$ and $\left(\mathcal{A}_{\mathrm{Y}} \mathbb{E}\right) \cap\left(\mathcal{B}_{\mathrm{Y}}, \mathbb{E}\right)=\Phi$,

where $\left(\mathcal{A}_{Y^{*}} \mathbb{E}\right)_{s}\left(\mathcal{B}_{Y^{*}} \mathbb{E}\right) \in \mathrm{S}, \delta^{*}, \mathrm{O}(X)_{Y}$.

Thus $\mathbb{Y}$ is a soft $-\delta^{*}-\mathrm{T}_{2}-$ space.

\section{4. soft $-\delta^{8}-$ regular, soft $-\delta^{8}-$ normal}

$$
\text { and soft }-\delta^{\varepsilon}-\mathrm{T}_{\mathrm{i}} ;(\mathrm{i}=3,4) \text { spaces }
$$

In this section, we define soft $-\delta^{*}-\mathrm{T}_{a}$ and soft $-\delta^{*}-\mathrm{T}_{4}$ spaces using ordinary points and characterize soft $-\delta^{*}-$ regular and soft $-\delta^{*}$-normal spaces.

Definition 4.1. Let $\left(X_{v}, \tau_{1}, \tau_{2}, \tau_{7,}, \mathbb{E}\right)$ be a soft-tritopological space over $\mathrm{X},\left(H_{x} \mathbb{E}\right)$ be a soft $-\delta^{*}-$ closed set in $X$, and $x \notin\left(H_{v} \mathbb{E}\right)$, if there exist a soft $-\delta^{*}-$ open sets $\left(F_{1 s} \mathbb{E}\right)$ and $\left(F_{2}, \mathbb{E}\right)$ such that " $x \in\left(F_{1 x} \mathbb{E}\right), \quad\left(H_{v} \mathbb{E}\right) \subseteq\left(F_{2 x} \mathbb{E}\right)$ and $\left(F_{1}, \mathbb{E}\right) \cap\left(F_{2}, \mathbb{E}\right)=\Phi "$. Then $\left(X_{v}, \tau_{1}, \tau_{2}, \tau_{y}, \mathbb{E}\right)$ is said to be soft $-\delta^{*}$-regular space.

Definition 4.2. A soft-tritopological space $\left(X_{v} \tau_{1}, \tau_{2}, \tau_{7}, \mathbb{E}\right)$ over $\mathrm{X}$ is said to be a soft $-\delta^{*}-\mathrm{T}_{a}-$ space iff it is a sof $t-\delta^{*}$-regular and soft $-\delta^{*}-\mathrm{T}_{1}-$ space.

Example 4.3. Let $\mathrm{X}=\left\{x_{1}, x_{2}\right\}$ be the universe set, and $\mathbb{E}=\left\{e_{1}, e_{2}\right\}$ be the set of parameters, Then $\left(\mathrm{X}_{v} \mathbb{E}\right)=\left\{\left(e_{1 v}\left\{x_{1}, x_{2}\right\}\right),\left(e_{2 v},\left\{x_{1}, x_{2}\right\}\right)\right\}$. By [5], its cardinality is defined by $|\mathcal{P}(X)|=2^{\left.\sum_{a s} \mid \mathcal{F}(\mathrm{e})\right]}$, where $|\mathcal{F}(\mathrm{e})|$ is the cardinality of $\mathcal{F}\left(\right.$ e) . (i.e. $|\mathcal{P}(X)|=2^{4}=16$ soft-sets).

Let $\left(x_{v} \tau_{1}, \mathbb{E}\right), \quad\left(x_{v} \tau_{2}, \mathbb{E}\right)$ and $\left(x_{v} \tau_{a}, \mathbb{E}\right)$ be the three soft-topological spaces on $X$, where their soft-topologies defined as follows:

$$
\begin{aligned}
& \tau_{1}=\left\{\Phi, x_{v}\left(M_{1}, \mathbb{E}\right),\left(\mathcal{M}_{2}, \mathbb{E}\right),\left(\mathcal{M}_{2}, \mathbb{E}\right)\right\}, \\
& \tau_{2}=\left\{\Phi_{x}, X_{x}\left(G_{1}, \mathbb{E}\right),\left(G_{2 x} \mathbb{E}\right),\left(G_{9 x}, \mathbb{E}\right),\left(G_{4}, \mathbb{E}\right)\right\} \\
& M_{1}\left(e_{1}\right)=\left\{x_{2}\right\} \quad, \quad M_{1}\left(e_{2}\right)=\mathrm{X} \\
& M_{2}\left(e_{1}\right)=\left\{x_{1}\right\} \quad, \quad M_{2}\left(e_{2}\right)=\left\{x_{2}\right\}
\end{aligned}
$$$$
\text { and }
$$$$
\tau_{\mathrm{a}}=\left\{\Phi_{0} x_{v}\left(\mathcal{H}_{1}, \mathbb{E}\right), \ldots,\left(\mathcal{H}_{10}, \mathbb{E}\right)\right\} \text {. Where the soft-open }
$$

$$
\begin{aligned}
& M_{a}\left(e_{1}\right)=\emptyset \quad, \quad M_{a}\left(e_{2}\right)=\left\{x_{2}\right\} \\
& \mathrm{G}_{1}\left(e_{1}\right)=\mathrm{X} \quad, \quad \mathrm{G}_{1}\left(e_{2}\right)=\emptyset \\
& \mathrm{G}_{2}\left(e_{1}\right)=\left\{x_{2}\right\} \quad, \quad \mathrm{G}_{2}\left(e_{2}\right)=\left\{x_{1}\right\} \\
& \mathrm{G}_{\mathrm{a}}\left(e_{1}\right)=\left\{x_{2}\right\} \quad, \quad \mathrm{G}_{\mathrm{a}}\left(e_{2}\right)=\emptyset \\
& \mathrm{G}_{4}\left(e_{1}\right)=\mathrm{X} \quad, \quad \mathrm{G}_{1}\left(e_{2}\right)=\left\{x_{1}\right\} \\
& \mathscr{H}_{1}\left(e_{1}\right)=\left\{x_{1}\right\} \quad, \quad \mathscr{H}_{1}\left(e_{2}\right)=\left\{x_{2}\right\} \\
& \mathscr{H}_{2}\left(e_{1}\right)=\mathrm{X} \quad, \quad \mathscr{H}_{2}\left(e_{2}\right)=\left\{x_{1}\right\} \\
& \mathscr{H}_{a}\left(e_{1}\right)=\left\{x_{1}\right\} \quad, \quad \mathscr{H}_{a}\left(e_{2}\right)=\emptyset \\
& \mathscr{H}_{4}\left(e_{1}\right)=\left\{x_{2}\right\} \quad, \quad \mathscr{H}_{4}\left(e_{2}\right)=\mathrm{X} \\
& \mathscr{H}_{5}\left(e_{1}\right)=\left\{x_{2}\right\} \quad, \quad \mathscr{H}_{5}\left(e_{2}\right)=\left\{x_{1}\right\} \\
& \mathscr{H}_{6}\left(e_{1}\right)=\emptyset \quad, \quad \mathscr{H}_{6}\left(e_{2}\right)=\left\{x_{2}\right\} \\
& \mathscr{H}_{7}\left(e_{1}\right)=\emptyset \quad, \quad \mathscr{H}_{7}\left(e_{2}\right)=\mathrm{X} \\
& \mathscr{H}_{\mathrm{g}}\left(e_{1}\right)=\emptyset \quad, \quad \mathscr{H}_{\mathrm{g}}\left(e_{2}\right)=\left\{x_{1}\right\} \\
& \mathscr{H}_{9}\left(e_{1}\right)=\left\{x_{1}\right\} \quad, \quad \mathscr{H}_{9}\left(\epsilon_{2}\right)=\mathrm{X} \\
& \mathscr{H}_{10}\left(e_{1}\right)=\left\{x_{1}\right\} \quad, \quad \mathscr{K}_{10}\left(e_{2}\right)=\left\{x_{1}\right\}
\end{aligned}
$$

The complement of the soft-open sets of soft-topology $\tau_{2}$ Are ;

$$
\begin{array}{lll}
\mathrm{G}_{1} \mathrm{C}\left(e_{1}\right)=\emptyset & , & \mathrm{G}_{1} \mathrm{C}\left(e_{2}\right)=\mathrm{X} \\
\mathrm{G}_{2} \mathrm{C}\left(e_{1}\right)=\left\{x_{1}\right\} & , & \mathrm{G}_{2}{ }^{\mathrm{C}}\left(e_{2}\right)=\left\{x_{2}\right\} \\
\mathrm{G}_{a} \mathrm{C}\left(e_{1}\right)=\left\{x_{1}\right\} & , & \mathrm{G}_{a}{ }^{\mathrm{C}}\left(e_{2}\right)=\mathrm{X} \\
\mathrm{G}_{4} \mathrm{C}\left(e_{1}\right)=\emptyset & , & \mathrm{G}_{4}{ }^{\mathrm{C}}\left(e_{2}\right)=\left\{x_{2}\right\}
\end{array}
$$

Hence the family of all soft- $\delta^{*}$-open sets of the soft-tritopological space $\left(X_{v} \tau_{1,}, \tau_{2}, \tau_{\mathrm{g}}, \mathbb{E}\right) \quad$ is,

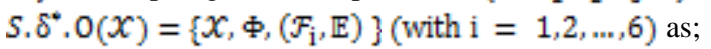

$$
\begin{array}{lll}
\mathcal{F}_{1}\left(e_{1}\right)=\left\{x_{1}\right\} & , & \mathcal{F}_{1}\left(e_{2}\right)=\left\{x_{2}\right\} \\
\mathcal{F}_{2}\left(e_{1}\right)=\left\{x_{1}\right\} & , & \mathcal{F}_{2}\left(e_{2}\right)=\emptyset \\
\mathcal{F}_{2}\left(e_{1}\right)=\left\{x_{2}\right\} & , & \mathcal{F}_{1}\left(e_{2}\right)=\left\{x_{1}\right\} \\
\mathcal{F}_{4}\left(e_{1}\right)=\left\{x_{2}\right\} & , & \mathcal{F}_{4}\left(e_{2}\right)=\mathrm{X} \\
\mathcal{F}_{5}\left(e_{1}\right)=\emptyset & & \mathcal{F}_{5}\left(e_{2}\right)=\left\{x_{2}\right\} \\
\mathcal{F}_{6}\left(e_{1}\right)=\mathrm{X} & & \mathcal{F}_{6}\left(e_{2}\right)=\left\{x_{1}\right\}
\end{array}
$$

Hence the family of all soft- $\delta^{*}-$ closed sets of the soft-tritopological space $\left(X_{v} \tau_{1,}, \tau_{2}, \tau_{g}, \mathbb{E}\right) \quad$ is,

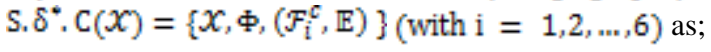

$$
\begin{array}{lll}
\mathcal{F}_{1}^{C}\left(e_{1}\right)=\left\{x_{2}\right\} & , & \mathcal{F}_{1}^{C}\left(e_{2}\right)=\left\{x_{1}\right\} \\
\mathcal{F}_{2}^{C}\left(e_{1}\right)=\left\{x_{2}\right\} & , & \mathcal{F}_{2}^{C}\left(e_{2}\right)=\mathrm{X} \\
\mathcal{F}_{9}^{C}\left(e_{1}\right)=\left\{x_{1}\right\} & , & \mathcal{F}_{1}^{C}\left(e_{2}\right)=\left\{x_{2}\right\} \\
\mathcal{F}_{4}^{C}\left(e_{1}\right)=\left\{x_{1}\right\} & , & \mathcal{F}_{4}^{C}\left(e_{2}\right)=\emptyset \\
\mathcal{F}_{5}^{C}\left(e_{1}\right)=\mathrm{X} & , & \mathcal{F}_{5}^{C}\left(e_{2}\right)=\left\{x_{1}\right\} \\
\mathcal{F}_{6}^{C}\left(e_{1}\right)=\emptyset & & \mathcal{F}_{6}^{C}\left(e_{2}\right)=\left\{x_{2}\right\}
\end{array}
$$


Let $x_{1}, x_{2} \in \mathrm{X}$ such that $x_{1} \neq x_{2}$

$\exists\left(\mathcal{F}_{6 *}^{\mathrm{C}} \mathbb{E}\right) \in \mathrm{S}, \delta^{*}{ }_{*} \mathrm{C}(X)$

$x_{1} \notin\left(\mathcal{F}_{6}^{\mathrm{C}}, \mathbb{E}\right)$

$\exists\left(\mathcal{F}_{6^{x}} \mathbb{E}\right),\left(\mathcal{F}_{5^{x}} \mathbb{E}\right) \in \mathrm{S}_{x} \delta^{*}{ }_{x} \mathrm{O}(X)$ such that

$x_{1} \in\left(\mathcal{F}_{6}, \mathbb{E}\right), \quad\left(\mathcal{F}_{6}^{\mathbb{C}} \mathbb{E}\right) \subseteq\left(\mathcal{F}_{5}, \mathbb{E}\right)$

$\left(\mathcal{F}_{6^{x}} \mathbb{E}\right) \cap\left(\mathcal{F}_{5^{x}} \mathbb{E}\right)=\Phi$, and so on $\ldots$

Thus $\left(X_{v} \tau_{1}, \tau_{2}, \tau_{g}, \mathbb{E}\right)$ is a soft $-\delta^{*}$-regular space.

Let $x_{1}, x_{2} \in \mathrm{X}$ such that $x_{1} \neq x_{2}$

$\exists\left(\mathcal{F}_{4}, \mathbb{E}\right)_{s}\left(\mathcal{F}_{6}, \mathbb{E}\right) \in S_{*} \delta^{*}, O(X)$ such that

$x_{1} \in\left(\mathcal{F}_{6}, \mathbb{E}\right){ }_{0} x_{2} \notin\left(\mathcal{F}_{6}, \mathbb{E}\right)$ and

$x_{2} \in\left(\mathcal{F}_{4}, \mathbb{E}\right), x_{1} \notin\left(\mathcal{F}_{4}, \mathbb{E}\right)$

Thus $\left(x_{v} \tau_{1}, \tau_{2}, \tau_{g}, \mathbb{E}\right)$ is a soft $-\delta^{*}-\mathrm{T}_{1}$-space.

Therefore $\left(x_{v} \tau_{1}, \tau_{2}, \tau_{7 x}, \mathbb{E}\right)$ is a soft $-\delta^{*}-\mathbb{T}_{2}-$ space.

Remark 4.4. (1) A soft $-\delta^{*}-T_{a}-$ space may not be a soft $-\delta^{*}-\mathrm{T}_{2}-$ space.

(2) If $\left(x_{v}, \tau_{1}, \tau_{2}, \tau_{a}, \mathbb{E}\right)$ is a soft $-\delta^{*}-T_{a}-$ space then $\left(\mathrm{X}_{x} \delta^{*} \cdot \tau_{\mathbb{E}}\right)$ may not be a $\mathrm{T}_{a}$-space for every parameter $\mathrm{e} \in \mathbb{E}$.

Example 4.5. Let $\mathrm{X}=\left\{x_{1}, x_{2}, x_{\mathrm{a}}\right\}$ be the universe set and $\mathbb{E}=\left\{e_{1}, e_{2}\right\}$ be the set of parameters, Then (the cardinality $|\mathcal{P}(x)|=2^{6}=64$ soft - set $)$.

And $\quad\left(x_{v} \tau_{1}, \mathbb{E}\right), \quad\left(x_{v} \tau_{2}, \mathbb{E}\right)$ and $\quad\left(x_{v}, \tau_{2}, \mathbb{E}\right)$ be three soft-topological spaces on $x$, and their soft-topologies defined as follows:

$\tau_{1}=\left\{\Phi_{s} x_{v}\left(M_{1}, \mathbb{E}\right),\left(M_{2}, \mathbb{E}\right),\left(\mathcal{M}_{2}, \mathbb{E}\right)\right\}$

$\tau_{2}=\left\{\Phi_{v}, X_{v}\left(G_{1}, \mathbb{E}\right),\left(G_{2 x} \mathbb{E}\right),\left(G_{a v} \mathbb{E}\right)\right\}$ and

$\tau_{\mathrm{a}}=\left\{\Phi_{v} x_{v}\left(\mathcal{H}_{1}, \mathrm{E}\right),\left(\mathcal{H}_{2}, \mathrm{E}\right), \ldots,\left(\mathcal{H}_{\mathrm{a} 0}, \mathrm{E}\right)\right\}$.

Where

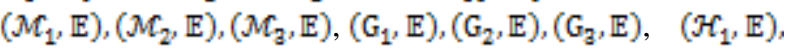

$\left(\mathcal{H}_{2,} \mathbb{E}\right)_{v \ldots s}\left(\mathcal{H}_{\mathrm{ag},} \mathbb{E}\right)$ are soft - open sets in $X$, defined as;

\begin{tabular}{|c|c|c|}
\hline$M_{1}\left(e_{1}\right)=X$ & , & $M_{1}\left(e_{2}\right)=\left\{x_{1}\right\}$ \\
\hline$M_{2}\left(e_{1}\right)=\mathrm{X}$ & , & $M_{2}\left(e_{2}\right)=\left\{x_{2}, x_{2}\right\}$ \\
\hline$M_{a}\left(e_{1}\right)=X$ & , & $M_{a}\left(e_{2}\right)=\emptyset$ \\
\hline $\mathrm{G}_{1}\left(e_{1}\right)=\emptyset$ & , & $\mathrm{G}_{1}\left(e_{2}\right)=\mathrm{X}$ \\
\hline $\mathrm{G}_{2}\left(e_{1}\right)=\emptyset$ & , & $\mathrm{G}_{2}\left(e_{2}\right)=\left\{x_{2}, x_{2}\right\}$ \\
\hline $\mathrm{G}_{a}\left(e_{1}\right)=\emptyset$ & , & $\mathrm{G}_{2}\left(e_{2}\right)=\left\{x_{1}\right\}$ \\
\hline $\mathscr{H}_{1}\left(e_{1}\right)=\mathrm{X}$ & , & $\mathscr{H}_{1}\left(\epsilon_{2}\right)=\emptyset$ \\
\hline $\mathscr{H}_{2}\left(e_{1}\right)=\left\{x_{1}\right\}$ & 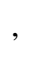 & $\mathscr{H}_{2}\left(e_{2}\right)=\emptyset$ \\
\hline $\mathscr{H}_{a}\left(e_{1}\right)=\left\{x_{2}\right\}$ & , & $\mathscr{H}_{a}\left(\epsilon_{2}\right)=\emptyset$ \\
\hline $\mathscr{H}_{4}\left(e_{1}\right)=\left\{x_{2}\right\}$ & , & $\mathscr{H}_{4}\left(e_{2}\right)=\emptyset$ \\
\hline $\mathscr{H}_{5}\left(e_{1}\right)=\left\{x_{1}, x_{2}\right\}$ & , & $\mathscr{H}_{5}\left(\epsilon_{2}\right)=\emptyset$ \\
\hline $\mathscr{H}_{6}\left(e_{1}\right)=\left\{x_{1}, x_{2}\right\}$ & 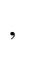 & $\mathscr{H}_{6}\left(\theta_{2}\right)=\emptyset$ \\
\hline $\mathscr{H}_{7}\left(e_{1}\right)=\left\{x_{2}, x_{\mathrm{g}}\right\}$ & , & $\mathscr{H}_{7}\left(\epsilon_{2}\right)=\emptyset$ \\
\hline
\end{tabular}

$$
\begin{aligned}
& \mathscr{H}_{g}\left(e_{1}\right)=\mathrm{X} \\
& \mathscr{H}_{\mathrm{g}}\left(e_{2}\right)=\left\{x_{1}\right\} \\
& \mathscr{H}_{9}\left(e_{1}\right)=\left\{x_{1}\right\} \\
& \mathscr{H}_{9}\left(e_{2}\right)=\left\{x_{1}\right\} \\
& \mathscr{H}_{10}\left(e_{1}\right)=\left\{x_{2}\right\} \\
& \mathscr{H}_{10}\left(e_{2}\right)=\left\{x_{1}\right\} \\
& \mathscr{H}_{11}\left(e_{1}\right)=\left\{x_{a}\right\} \\
& \mathscr{H}_{11}\left(\epsilon_{2}\right)=\left\{x_{1}\right\} \\
& \mathscr{H}_{12}\left(e_{1}\right)=\left\{x_{1}, x_{2}\right\} \quad, \quad \mathscr{H}_{12}\left(e_{2}\right)=\left\{x_{1}\right\} \\
& \mathscr{H}_{13}\left(e_{1}\right)=\left\{x_{1}, x_{2}\right\} \quad, \quad \mathscr{H}_{1 a}\left(e_{2}\right)=\left\{x_{1}\right\} \\
& \mathscr{H}_{14}\left(e_{1}\right)=\left\{x_{2}, x_{a}\right\} \quad, \quad \mathscr{H}_{14}\left(e_{2}\right)=\left\{x_{1}\right\} \\
& \mathscr{H}_{15}\left(e_{1}\right)=\emptyset \\
& \mathscr{H}_{16}\left(e_{1}\right)=\mathrm{X} \\
& \mathscr{H}_{15}\left(e_{2}\right)=\left\{x_{1}\right\} \\
& \mathscr{H}_{17}\left(e_{1}\right)=\left\{x_{1}\right\} \\
& \mathscr{K}_{16}\left(e_{2}\right)=\left\{x_{2}, x_{2}\right\} \\
& \mathscr{H}_{18}\left(e_{1}\right)=\left\{x_{2}\right\} \\
& \mathscr{H}_{17}\left(e_{2}\right)=\left\{x_{2}, x_{2}\right\} \\
& \mathscr{H}_{19}\left(e_{1}\right)=\left\{x_{2}\right\} \\
& \mathscr{H}_{18}\left(\epsilon_{2}\right)=\left\{x_{2}, x_{2}\right\} \\
& \mathscr{H}_{20}\left(e_{1}\right)=\left\{x_{1}, x_{2}\right\} \\
& \mathscr{H}_{19}\left(e_{2}\right)=\left\{x_{2}, x_{2}\right\} \\
& \mathscr{H}_{21}\left(e_{1}\right)=\left\{x_{1}, x_{2}\right\} \\
& \mathscr{H}_{20}\left(e_{2}\right)=\left\{x_{2}, x_{a}\right\} \\
& \mathscr{H}_{22}\left(e_{1}\right)=\left\{x_{2}, x_{2}\right\} \\
& \mathscr{H}_{21}\left(e_{2}\right)=\left\{x_{2}, x_{2}\right\} \\
& \mathscr{H}_{2 a}\left(e_{1}\right)=\emptyset \\
& \mathscr{H}_{22}\left(e_{2}\right)=\left\{x_{2}, x_{a}\right\} \\
& \mathscr{H}_{24}\left(e_{1}\right)=\left\{x_{1}\right\} \\
& \mathscr{H}_{2 a}\left(e_{2}\right)=\left\{x_{2}, x_{a}\right\} \\
& \mathscr{H}_{25}\left(e_{1}\right)=\left\{x_{2}\right\} \\
& \mathscr{H}_{24}\left(e_{2}\right)=\mathrm{X} \\
& \mathscr{H}_{26}\left(e_{1}\right)=\left\{x_{2}\right\} \\
& \mathscr{H}_{25}\left(\epsilon_{2}\right)=\mathrm{X} \\
& \mathscr{H}_{27}\left(e_{1}\right)=\left\{x_{1}, x_{2}\right\} \\
& \mathscr{H}_{26}\left(e_{2}\right)=\mathrm{X} \\
& \mathscr{H}_{2 \mathrm{~g}}\left(e_{1}\right)=\left\{x_{1}, x_{\mathrm{a}}\right\} \\
& \mathscr{H}_{27}\left(\epsilon_{2}\right)=\mathrm{X} \\
& \mathscr{H}_{2 \mathrm{~g}}\left(\epsilon_{2}\right)=\mathrm{X} \\
& \mathscr{H}_{29}\left(e_{1}\right)=\left\{x_{2}, x_{2}\right\} \\
& \mathscr{K}_{29}\left(e_{2}\right)=\mathrm{X} \\
& H_{a 0}\left(e_{1}\right)=\emptyset \\
& \mathscr{H}_{a 0}\left(e_{2}\right)=\mathrm{X}
\end{aligned}
$$

Hence the family of all soft- $\delta^{*}$-open sets of the soft-tritopological space $\left(X_{v} \tau_{1}, \tau_{2}, \tau_{z}, \mathbb{E}\right) \quad$ is, S. $\delta^{*} \mathrm{O}(X)=\left\{X_{v} \Phi_{v}\left(\mathcal{F}_{\mathrm{i},} \mathbb{E}\right)\right\}$ (with $\left.\mathrm{i}=1,2_{v} \ldots, 30\right)$ defined as;

$$
\begin{array}{lll}
\mathcal{F}_{1}\left(e_{1}\right)=\mathrm{X} & , & \mathcal{F}_{1}\left(e_{2}\right)=\emptyset \\
\mathcal{F}_{2}\left(e_{1}\right)=\left\{x_{1}\right\} & , & \mathcal{F}_{2}\left(e_{2}\right)=\emptyset \\
\mathcal{F}_{2}\left(e_{1}\right)=\left\{x_{2}\right\} & , & \mathcal{F}_{2}\left(e_{2}\right)=\emptyset \\
\mathcal{F}_{4}\left(e_{1}\right)=\left\{x_{2}\right\} & , & \mathcal{F}_{4}\left(e_{2}\right)=\emptyset \\
\mathcal{F}_{5}\left(e_{1}\right)=\left\{x_{1}, x_{2}\right\} & , & \mathcal{F}_{5}\left(e_{2}\right)=\emptyset \\
\mathcal{F}_{6}\left(e_{1}\right)=\left\{x_{1}, x_{2}\right\} & , & \mathcal{F}_{6}\left(e_{2}\right)=\emptyset \\
\mathcal{F}_{7}\left(e_{1}\right)=\left\{x_{2}, x_{2}\right\} & , & \mathcal{F}_{7}\left(e_{2}\right)=\emptyset \\
\mathcal{F}_{9}\left(e_{1}\right)=\mathrm{X} & , & \mathcal{F}_{2}\left(e_{2}\right)=\left\{x_{1}\right\} \\
\mathcal{F}_{9}\left(e_{1}\right)=\left\{x_{1}\right\} & , & \mathcal{F}_{9}\left(e_{2}\right)=\left\{x_{1}\right\} \\
\mathcal{F}_{10}\left(e_{1}\right)=\left\{x_{2}\right\} & , & \mathcal{F}_{10}\left(e_{2}\right)=\left\{x_{1}\right\} \\
\mathcal{F}_{11}\left(e_{1}\right)=\left\{x_{2}\right\} & , & \mathcal{F}_{11}\left(e_{2}\right)=\left\{x_{1}\right\} \\
\mathcal{F}_{12}\left(e_{1}\right)=\left\{x_{1}, x_{2}\right\} & , & \mathcal{F}_{12}\left(e_{2}\right)=\left\{x_{1}\right\}
\end{array}
$$




$$
\begin{aligned}
& \mathcal{F}_{1 \mathrm{a}}\left(e_{1}\right)=\left\{x_{1}, x_{2}\right\} \quad, \quad \mathcal{F}_{1 \mathrm{a}}\left(e_{2}\right)=\left\{x_{1}\right\} \\
& \mathcal{F}_{14}\left(e_{1}\right)=\left\{x_{2}, x_{1}\right\} \quad, \quad \mathcal{F}_{14}\left(e_{2}\right)=\left\{x_{1}\right\} \\
& \mathcal{F}_{15}\left(e_{1}\right)=\emptyset \quad, \quad \mathcal{F}_{15}\left(e_{2}\right)=\left\{x_{1}\right\} \\
& \mathcal{F}_{16}\left(e_{1}\right)=\mathrm{X} \\
& \mathcal{F}_{17}\left(e_{1}\right)=\left\{x_{1}\right\} \\
& \mathcal{F}_{18}\left(e_{1}\right)=\left\{x_{2}\right\} \\
& \mathcal{F}_{19}\left(e_{1}\right)=\left\{x_{2}\right\} \\
& \mathcal{F}_{20}\left(e_{1}\right)=\left\{x_{1}, x_{2}\right\} \\
& \mathcal{F}_{21}\left(e_{1}\right)=\left\{x_{1}, x_{2}\right\} \\
& \mathcal{F}_{22}\left(e_{1}\right)=\left\{x_{2}, x_{2}\right\} \\
& \mathcal{F}_{16}\left(e_{2}\right)=\left\{x_{2}, x_{2}\right\} \\
& \mathcal{F}_{17}\left(e_{2}\right)=\left\{x_{2}, x_{a}\right\} \\
& \mathcal{F}_{1 \mathrm{~g}}\left(e_{2}\right)=\left\{x_{2}, x_{\mathrm{a}}\right\} \\
& \mathcal{F}_{19}\left(e_{2}\right)=\left\{x_{2}, x_{2}\right\} \\
& \mathcal{F}_{20}\left(e_{2}\right)=\left\{x_{2}, x_{a}\right\} \\
& \mathcal{F}_{21}\left(e_{2}\right)=\left\{x_{2}, x_{2}\right\} \\
& \mathcal{F}_{2 a}\left(e_{1}\right)=\emptyset \\
& \mathcal{F}_{24}\left(e_{1}\right)=\left\{x_{1}\right\} \\
& \mathcal{F}_{25}\left(e_{1}\right)=\left\{x_{2}\right\} \\
& \mathcal{F}_{26}\left(e_{1}\right)=\left\{x_{2}\right\} \\
& \mathcal{F}_{27}\left(e_{1}\right)=\left\{x_{1}, x_{2}\right\} \\
& \mathcal{F}_{22}\left(e_{2}\right)=\left\{x_{2}, x_{2}\right\} \\
& \mathcal{F}_{2 a}\left(e_{2}\right)=\left\{x_{2}, x_{2}\right\} \\
& \mathcal{F}_{24}\left(e_{2}\right)=\mathrm{X} \\
& \mathcal{F}_{25}\left(e_{2}\right)=\mathrm{X} \\
& \mathcal{F}_{26}\left(e_{2}\right)=\mathrm{X} \\
& \mathcal{F}_{28}\left(e_{1}\right)=\left\{x_{1}, x_{2}\right\} \\
& \mathcal{F}_{27}\left(e_{2}\right)=\mathrm{X} \\
& \mathcal{F}_{29}\left(e_{1}\right)=\left\{x_{2}, x_{2}\right\} \\
& \mathcal{F}_{2 \mathrm{~B}}\left(e_{2}\right)=\mathrm{X} \\
& \mathcal{F}_{\mathrm{a} 0}\left(e_{1}\right)=\emptyset \\
& \mathcal{F}_{29}\left(e_{2}\right)=\mathrm{X} \\
& \mathcal{F}_{\mathrm{ga}}\left(e_{2}\right)=\mathrm{X}
\end{aligned}
$$

Hence the family of all soft $-\delta^{*}$-closed sets of the soft-tritopological space $\left(X_{v} \tau_{1,} \tau_{2,} \tau_{\mathbb{Z}^{p}} \mathbb{E}\right) \quad$ is, S. $\delta^{*} \mathrm{C}(X)=\left\{X_{v} \Phi_{s}\left(\mathcal{F}_{\mathrm{i}}{ }^{c}{ }_{v} \mathbb{E}\right)\right\}$ (with $\left.\mathrm{i}=1,2_{v},{ }_{v} 30\right)$.

$$
\begin{aligned}
& F_{1}^{C}\left(e_{1}\right)=\emptyset \quad, \quad F_{1}^{C}\left(e_{2}\right)=\mathrm{X} \\
& \mathcal{F}_{2}^{C}\left(e_{1}\right)=\left\{x_{2}, x_{2}\right\} \quad, \quad \mathcal{F}_{2}^{C}\left(e_{2}\right)=\mathrm{X} \\
& \mathcal{F}_{a}^{c}\left(e_{1}\right)=\left\{x_{1}, x_{a}\right\} \quad, \quad \mathcal{F}_{a}^{c}\left(e_{2}\right)=\mathrm{X} \\
& \mathcal{F}_{4}^{\mathrm{C}}\left(e_{1}\right)=\left\{x_{1}, x_{2}\right\} \quad, \quad \mathcal{F}_{4}^{\mathrm{C}}\left(e_{2}\right)=\mathrm{X} \\
& \mathcal{F}_{5}^{c}\left(e_{1}\right)=\left\{x_{9}\right\} \quad, \quad \mathcal{F}_{5}^{c}\left(e_{2}\right)=\mathrm{X} \\
& F_{6}^{c}\left(e_{1}\right)=\left\{x_{2}\right\} \quad, \quad F_{6}^{c}\left(e_{2}\right)=\mathrm{X} \\
& \mathcal{F}_{7}^{C}\left(e_{1}\right)=\left\{x_{1}\right\} \quad, \quad \mathcal{F}_{7}^{C}\left(e_{2}\right)=\mathrm{X} \\
& \mathcal{F}_{\mathrm{g}}^{\mathrm{C}}\left(e_{1}\right)=\emptyset \quad, \quad \mathcal{F}_{\mathrm{g}}^{\mathcal{C}}\left(e_{2}\right)=\left\{x_{2}, x_{\mathrm{a}}\right\} \\
& \mathcal{F}_{9}{ }^{C}\left(e_{1}\right)=\left\{x_{2}, x_{2}\right\} \quad, \quad \mathcal{F}_{9}^{C}\left(e_{2}\right)=\left\{x_{2}, x_{2}\right\} \\
& \mathcal{F}_{10}^{\mathscr{C}}\left(e_{1}\right)=\left\{x_{1}, x_{2}\right\} \quad, \quad \mathcal{F}_{10}^{\mathscr{C}}\left(e_{2}\right)=\left\{x_{2}, x_{a}\right\} \\
& \mathcal{F}_{11}^{C}\left(e_{1}\right)=\left\{x_{1}, x_{2}\right\} \quad, \quad \mathcal{F}_{11}^{C}\left(e_{2}\right)=\left\{x_{2}, x_{2}\right\} \\
& \mathcal{F}_{12}^{c}\left(e_{1}\right)=\left\{x_{2}\right\} \quad, \quad \mathcal{F}_{12}^{c}\left(e_{2}\right)=\left\{x_{2}, x_{2}\right\} \\
& \mathcal{F}_{1 \mathrm{a}}^{C}\left(e_{1}\right)=\left\{x_{2}\right\} \quad, \quad \mathcal{F}_{1 \mathrm{a}}^{C}\left(e_{2}\right)=\left\{x_{2}, x_{\mathrm{a}}\right\} \\
& \mathcal{F}_{14}^{C}\left(e_{1}\right)=\left\{x_{1}\right\} \quad, \quad \mathcal{F}_{14}^{C}\left(e_{2}\right)=\left\{x_{2}, x_{2}\right\} \\
& \mathcal{F}_{15}^{C}\left(e_{1}\right)=\mathrm{X} \quad, \quad \mathcal{F}_{15}^{C}\left(e_{2}\right)=\left\{x_{2}, x_{a}\right\} \\
& \mathcal{F}_{16}^{C}\left(e_{1}\right)=\emptyset \quad, \quad \mathcal{F}_{16}^{C}\left(e_{2}\right)=\left\{x_{1}\right\} \\
& \mathcal{F}_{17}^{c}\left(e_{1}\right)=\left\{x_{2}, x_{2}\right\} \quad, \quad \mathcal{F}_{17}^{c}\left(e_{2}\right)=\left\{x_{1}\right\}
\end{aligned}
$$

$$
\begin{aligned}
& \mathcal{F}_{18}^{C}\left(e_{1}\right)=\left\{x_{1}, x_{2}\right\} \quad, \quad \mathcal{F}_{18}^{C}\left(e_{2}\right)=\left\{x_{1}\right\} \\
& \mathcal{F}_{19}^{C}\left(e_{1}\right)=\left\{x_{1}, x_{2}\right\} \quad, \quad \mathcal{F}_{19}^{C}\left(e_{2}\right)=\left\{x_{1}\right\} \\
& \mathcal{F}_{20}^{C}\left(e_{1}\right)=\left\{x_{2}\right\} \quad, \quad \mathcal{F}_{20}^{\mathscr{C}}\left(e_{2}\right)=\left\{x_{1}\right\} \\
& \mathcal{F}_{21}^{C}\left(e_{1}\right)=\left\{x_{2}\right\} \quad, \quad \mathcal{F}_{21}^{C}\left(e_{2}\right)=\left\{x_{1}\right\} \\
& \mathcal{F}_{22}^{C}\left(e_{1}\right)=\left\{x_{1}\right\} \quad, \quad \mathcal{F}_{22}^{C}\left(e_{2}\right)=\left\{x_{1}\right\} \\
& \mathcal{F}_{2 \mathrm{a}}^{C}\left(e_{1}\right)=\mathrm{X} \quad, \quad \mathcal{F}_{2 \mathrm{a}}^{\mathrm{C}}\left(e_{2}\right)=\left\{x_{1}\right\} \\
& \mathcal{F}_{24}{ }^{C}\left(e_{1}\right)=\left\{x_{2}, x_{2}\right\} \quad, \quad \mathcal{F}_{24}^{C}\left(e_{2}\right)=\emptyset \\
& \mathcal{F}_{25}^{\mathcal{C}}\left(e_{1}\right)=\left\{x_{1}, x_{2}\right\} \quad, \quad \mathcal{F}_{25}^{C}\left(e_{2}\right)=\emptyset \\
& \mathcal{F}_{26}^{C}\left(e_{1}\right)=\left\{x_{1}, x_{2}\right\} \quad, \quad \mathcal{F}_{26}^{C}\left(e_{2}\right)=\emptyset \\
& \mathcal{F}_{27}^{C}\left(e_{1}\right)=\left\{x_{2}\right\} \quad, \quad \mathcal{F}_{27}^{\mathcal{C}}\left(e_{2}\right)=\emptyset \\
& \mathcal{F}_{2 \mathrm{~g}}^{C}\left(e_{1}\right)=\left\{x_{2}\right\} \quad, \quad \mathcal{F}_{2 \mathrm{~g}}^{C}\left(e_{2}\right)=\emptyset \\
& \mathcal{F}_{29}^{c}\left(e_{1}\right)=\left\{x_{1}\right\} \quad, \quad \mathcal{F}_{29}^{c}\left(e_{2}\right)=\emptyset \\
& \mathcal{F}_{\mathrm{ab}}^{\mathrm{C}}\left(e_{1}\right)=\mathrm{X} \quad, \quad \mathcal{F}_{\mathrm{aj}}^{\mathrm{C}}\left(e_{2}\right)=\emptyset
\end{aligned}
$$

We note that $\left(X_{v}, \tau_{1}, \tau_{2}, \tau_{2}, \mathbb{E}\right)$ is a soft $-\delta^{*}-\mathbb{T}_{a}$-space but it is not a soft $-\delta^{*}-T_{2}-$ space because $x_{2}, x_{a} \in X$ but there do not exist soft $-\hat{\delta}^{*}$-open sets $\left(\mathrm{F}_{x} \mathrm{E}\right)$ and $\left(\mathrm{G}_{*} \mathrm{E}\right)$ such that $x_{2} \in\left(F_{x} E\right), x_{a} \in\left(G_{s} E\right)$ and $(F, E) \cap(G, E)=\Phi$. Thus every soft $-\delta^{*}-T_{a}-$ space is not necessarily a soft $-\delta^{*}-T_{2}-$ space. Now we have,

$\delta^{*}, \tau_{e_{1}}=\left\{\emptyset, \mathrm{X}_{v}\left\{x_{1}\right\},\left\{x_{2}\right\},\left\{x_{2}\right\},\left\{x_{1}, x_{2}\right\},\left\{x_{1}, x_{2}\right\},\left\{x_{2}, x_{2}\right\}\right\}$ and $\delta^{*} \cdot \tau_{g_{2}}=\left\{\emptyset, X_{v}\left\{x_{1}\right\},\left\{x_{2}, x_{2}\right\}\right\}$

Here $\left(\mathrm{X}_{v} \delta^{*} \cdot \mathrm{\tau}_{\sigma_{2}}\right)$ is not a $\mathrm{T}_{a}$-space. This shows that if $\left(X_{v}, \tau_{1}, \tau_{2}, \tau_{g}, \mathbb{E}\right)$ is a soft $-\delta^{*}-\mathrm{T}_{a}$-space then $\left(\mathrm{X}_{v} \delta^{*}, \tau_{\mathrm{e}}\right)$ may not be a $T_{a}$-space for every parameter $e \in \mathbb{E}$,

Proposition 4.6. Let $\left(X_{x}, \tau_{1}, \tau_{2}, \tau_{3}, \mathbb{E}\right)$ be a soft-tritopological space over $\mathrm{X}$ and $\mathrm{Y}$ be a non-empty subset of $\mathrm{X}$. If $x$ is a soft $-\delta^{*}-\mathrm{T}_{a}-$ space then the soft-subspace $Y$ is a soft $-\delta^{*}-T_{a}-$ space.

Proof. By proposition 3.9. the soft-subspace $Y$ is a soft $-\delta^{*}-\mathrm{T}_{1}$-space. Let $y \in \mathrm{Y}$ and $\left(\mathrm{F}_{0} \mathrm{E}\right)$ be a soft $-\delta^{*}-$ closed set in $Y$ such that $y \notin\left(F_{0} \mathrm{E}\right)$.

Then $y \notin\left(\left(F_{x} E\right) \cap\left(G_{v} E\right)\right)$. where $\left(F_{s} E\right)=\left(\left(Y_{x} E\right) \cap\left(G_{x} E\right)\right)$. for some soft $-\delta^{*}-$ closed set in $X$, by theorem 3.13. in [16]. But $y \in\left(\mathrm{Y}_{\mathrm{g}} \mathrm{E}\right)$, so $y \notin\left(\mathrm{G}_{x} \mathrm{E}\right)$. As $x$ is a soft $-\delta^{*}-T_{a}-$ space, so there exist soft $-\delta^{*}$ - open sets $\left(\mathrm{G}_{1}, E\right)$ and $\left(\mathrm{G}_{2,}, E\right)$ in $\mathrm{X}$ such that $y \in\left(\mathrm{G}_{1}, E\right)$, $\left(\mathrm{G}_{x} \mathrm{E}\right) \subseteq\left(\mathrm{G}_{2}, E\right)$ and $\left(\mathrm{G}_{1}, E\right) \cap\left(\mathrm{G}_{2}, E\right)=\Phi$. Now if we take;

$\left(F_{1 x} E\right)=\left(\left(Y_{x} E\right) \cap\left(G_{1 x} E\right)\right)$ and $\left(F_{2 x} E\right)=\left(\left(Y_{x} E\right) \cap\left(G_{2 x} E\right)\right)$ , then $\left(F_{1}, E\right)$ and $\left(F_{2}, E\right) \in S_{x} \delta^{*}, O(X)_{Y}$ such that $y \in\left(F_{1}, E\right)$ $\left(F_{v} E\right) \subseteq\left(Y_{x} E\right) \cap\left(G_{2}, E\right)=\left(F_{2}, E\right) \quad$ and $\left(F_{1}, E\right) \cap\left(F_{2}, E\right) \subseteq\left(G_{1}, E\right) \cap\left(G_{2}, E\right)=\Phi$, $\left(F_{1}, E\right) \cap\left(F_{2}, E\right)=\Phi$.

Thus the soft-subspace $\Psi$ is a soft $-\delta^{*}-T_{a}-$ space.

Definition 4.7. Let $\left(x_{v} \tau_{1}, \tau_{2}, \tau_{3}, \mathbb{E}\right)$ be a soft-tritopological space over $\mathrm{X},\left(H_{v} \mathbb{E}\right)$ and $\left(G_{v} \mathbb{E}\right)$ soft $-\delta^{*}-$ closed sets 
in $\mathrm{X}$ such that $\left(H_{v} \mathbb{E}\right) \cap\left(G_{v} \mathbb{E}\right)=\Phi$, If there exist soft $-\delta^{*}$ - open sets $\left(F_{1,} \mathbb{E}\right)$ and $\left(F_{2}, \mathbb{E}\right)$ such that

“( $\left.H_{v} \mathbb{E}\right) \subseteq\left(F_{1 x} \mathbb{E}\right),\left(G_{v} \mathbb{E}\right) \subseteq\left(F_{2}, \mathbb{E}\right) \operatorname{and}\left(F_{10} \mathbb{E}\right) \cap\left(F_{2 v} \mathbb{E}\right)=\Phi "$. Then $\left(X_{v}, \tau_{10}, \tau_{2}, \tau_{2}, \mathbb{E}\right)$ is called a soft $-\delta^{*}$-normal space.

Definition 4.8. A soft-tritopological space $\left(X_{v}, \tau_{1}, \tau_{2}, \tau_{7 x}, \mathbb{E}\right)$ over $X$ is said to be a soft $-\delta^{*}-T_{4}$-space iff it is a soft $-\delta^{*}$-normal and soft $-\delta^{*}-T_{1}-$ space.

Example 4.9. Let $\mathrm{X}=\left\{x_{1}, x_{2}\right\}$ be the universe set and $\mathbb{E}=\left\{e_{1}, e_{2}\right\}$ be the set of parameters, Then $\left(x_{v} \mathbb{E}\right)=\left\{\left(e_{1,}\left\{x_{1}, x_{2}\right\}\right),\left(e_{2},\left\{x_{1}, x_{2}\right\}\right)\right\}$. By [5], its cardinality is defined by $\left.|\mathcal{P}(X)|=2^{\sum_{\kappa: B} \mid}|\mathcal{F}(\mathrm{e})|\right]$, where $|\mathcal{F}(\mathrm{e})|$ is the cardinality of $\mathcal{F}(\mathrm{e})$. (i.e. $|\mathcal{P}(X)|=2^{4}=16$ soft-set).

Let $\left(X_{v} \tau_{1,} \mathbb{E}\right),\left(X_{v} \tau_{2}, \mathbb{E}\right)$ and $\left(X_{v} \tau_{a x} \mathbb{E}\right)$ be the three soft $t$-topological spaces on $X$, where their soft-topologies defined as follows:

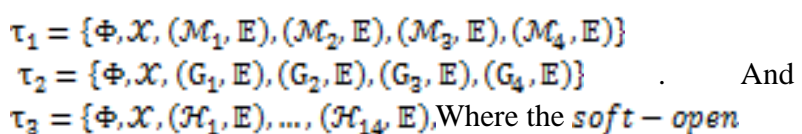

sets over $X$ in three soft-topologies, defined as follows:

\begin{tabular}{|c|c|c|}
\hline$M_{1}\left(e_{1}\right)=\left\{x_{1}\right\}$ & , & $M_{1}\left(e_{2}\right)=\mathrm{X}$ \\
\hline$M_{2}\left(e_{1}\right)=\left\{x_{1}\right\}$ & , & $\mathcal{M}_{2}\left(e_{2}\right)=\left\{x_{2}\right\}$ \\
\hline$M_{a}\left(e_{1}\right)=\emptyset$ & , & $M_{a}\left(e_{2}\right)=X$ \\
\hline$M_{4}\left(e_{1}\right)=\emptyset$ & , & $M_{4}\left(e_{2}\right)=\left\{x_{2}\right\}$ \\
\hline $\mathrm{G}_{1}\left(e_{1}\right)=\mathrm{X}$ & , & $\mathrm{G}_{1}\left(e_{2}\right)=\emptyset$ \\
\hline $\mathrm{G}_{2}\left(e_{1}\right)=\left\{x_{2}\right\}$ & , & $\mathrm{G}_{2}\left(e_{2}\right)=\left\{x_{1}\right\}$ \\
\hline $\mathrm{G}_{\mathrm{a}}\left(e_{1}\right)=\left\{x_{2}\right\}$ & , & $\mathrm{G}_{\mathrm{a}}\left(e_{2}\right)=\emptyset$ \\
\hline $\mathrm{G}_{4}\left(e_{1}\right)=\mathrm{X}$ & , & $\mathrm{G}_{4}\left(e_{2}\right)=\left\{x_{1}\right\}$ \\
\hline $\mathscr{H}_{1}\left(e_{1}\right)=\mathrm{X}$ & , & $\mathscr{H}_{1}\left(e_{2}\right)=\left\{x_{2}\right\}$ \\
\hline $\mathscr{H}_{2}\left(e_{1}\right)=\left\{x_{1}\right\}$ & , & $\mathscr{H}_{2}\left(e_{2}\right)=\mathrm{X}$ \\
\hline $\mathscr{H}_{a}\left(e_{1}\right)=\left\{x_{2}\right\}$ & , & $\mathscr{H}_{a}\left(e_{2}\right)=\emptyset$ \\
\hline $\mathscr{H}_{4}\left(e_{1}\right)=\left\{x_{2}\right\}$ & , & $\mathscr{H}_{4}\left(e_{2}\right)=\mathrm{X}$ \\
\hline $\mathscr{H}_{5}\left(e_{1}\right)=\left\{x_{1}\right\}$ & , & $\mathscr{H}_{5}\left(e_{2}\right)=\left\{x_{1}\right\}$ \\
\hline $\mathscr{H}_{6}\left(e_{1}\right)=\emptyset$ & , & $\mathscr{H}_{6}\left(e_{2}\right)=\left\{x_{1}\right\}$ \\
\hline $\mathscr{H}_{7}\left(e_{1}\right)=\left\{x_{1}\right\}$ & , & $\mathscr{H}_{7}\left(e_{2}\right)=\emptyset$ \\
\hline $\mathscr{H}_{8}\left(e_{1}\right)=\mathrm{X}$ & , & $\mathscr{H}_{2}\left(e_{2}\right)=\left\{x_{1}\right\}$ \\
\hline $\mathscr{H}_{9}\left(e_{1}\right)=\left\{x_{2}\right\}$ & , & $\mathscr{H}_{9}\left(e_{2}\right)=\left\{x_{1}\right\}$ \\
\hline $\mathscr{H}_{10}\left(e_{1}\right)=\left\{x_{1}\right\}$ & 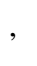 & $\mathscr{H}_{10}\left(e_{2}\right)=\left\{x_{2}\right\}$ \\
\hline $\mathscr{H}_{11}\left(e_{1}\right)=\left\{x_{2}\right\}$ & , & $\mathscr{H}_{11}\left(e_{2}\right)=\left\{x_{2}\right\}$ \\
\hline $\mathscr{H}_{12}\left(e_{1}\right)=\emptyset$ & , & $\mathscr{H}_{12}\left(e_{2}\right)=\left\{x_{2}\right\}$ \\
\hline $\mathscr{H}_{1 a}\left(e_{1}\right)=\mathrm{X}$ & , & $\mathscr{H}_{12}\left(e_{2}\right)=\emptyset$ \\
\hline $\mathscr{H}_{14}\left(e_{1}\right)=\emptyset$ & , & $\mathscr{H}_{14}\left(e_{2}\right)=\mathrm{X}$ \\
\hline
\end{tabular}

The complement of the soft-open sets of $\tau_{2}$ Are ;

$$
\begin{array}{lll}
\mathrm{G}_{1} \mathrm{C}\left(e_{1}\right)=\emptyset & , & \mathrm{G}_{1} \mathrm{C}\left(e_{2}\right)=\mathrm{X} \\
\mathrm{G}_{2} \mathrm{C}\left(e_{1}\right)=\left\{x_{1}\right\} & , & \mathrm{G}_{2}^{\mathrm{C}}\left(e_{2}\right)=\left\{x_{2}\right\} \\
\mathrm{G}_{a} \mathrm{C}\left(e_{1}\right)=\left\{x_{1}\right\} & , & \mathrm{G}_{2} \mathrm{C}\left(e_{2}\right)=\mathrm{X} \\
\mathrm{G}_{4} \mathrm{C}\left(e_{1}\right)=\emptyset & , & \mathrm{G}_{4} \mathrm{C}\left(e_{2}\right)=\left\{x_{2}\right\}
\end{array}
$$

Hence the family of all soft- $\delta^{*}$-open sets of the soft-tritopological space $\left(X_{v} \tau_{1}, \tau_{2}, \tau_{a}, \mathbb{E}\right) \quad$ is, S. $\delta^{*}, \mathrm{O}(X)=\left\{X_{v} \Phi_{s}\left(\mathcal{F}_{\mathrm{i}^{x}} \mathbb{E}\right)\right\}$ (with $\left.\mathrm{i}=1,2_{x, \ldots} 14\right)$ as;

$$
\begin{array}{lll}
\mathcal{F}_{1}\left(e_{1}\right)=\left\{x_{1}\right\} & , & \mathcal{F}_{1}\left(e_{2}\right)=\left\{x_{1}\right\} \\
\mathcal{F}_{2}\left(e_{1}\right)=\left\{x_{1}\right\} & , & \mathcal{F}_{2}\left(e_{2}\right)=\left\{x_{2}\right\} \\
\mathcal{F}_{2}\left(e_{1}\right)=\left\{x_{1}\right\} & , & \mathcal{F}_{2}\left(e_{2}\right)=\mathrm{X} \\
\mathcal{F}_{4}\left(e_{1}\right)=\left\{x_{1}\right\} & , & \mathcal{F}_{4}\left(e_{2}\right)=\emptyset \\
\mathcal{F}_{5}\left(e_{1}\right)=\left\{x_{2}\right\} & , & \mathcal{F}_{5}\left(e_{2}\right)=\left\{x_{2}\right\} \\
\mathcal{F}_{6}\left(e_{1}\right)=\left\{x_{2}\right\} & , & \mathcal{F}_{6}\left(e_{2}\right)=\left\{x_{1}\right\} \\
\mathcal{F}_{7}\left(e_{1}\right)=\left\{x_{2}\right\} & , & \mathcal{F}_{7}\left(e_{2}\right)=\mathrm{X} \\
\mathcal{F}_{9}\left(e_{1}\right)=\left\{x_{2}\right\} & , & \mathcal{F}_{9}\left(e_{2}\right)=\emptyset \\
\mathcal{F}_{9}\left(e_{1}\right)=\emptyset & , & \mathcal{F}_{9}\left(e_{2}\right)=\left\{x_{1}\right\} \\
\mathcal{F}_{10}\left(e_{1}\right)=\emptyset & , & \mathcal{F}_{10}\left(e_{2}\right)=\left\{x_{2}\right\} \\
\mathcal{F}_{11}\left(e_{1}\right)=\emptyset & , & \mathcal{F}_{11}\left(e_{2}\right)=\mathrm{X} \\
\mathcal{F}_{12}\left(e_{1}\right)=\mathrm{X} & , & \mathcal{F}_{12}\left(e_{2}\right)=\emptyset \\
\mathcal{F}_{19}\left(e_{1}\right)=\mathrm{X} & , & \mathcal{F}_{13}\left(e_{2}\right)=\left\{x_{1}\right\} \\
\mathcal{F}_{14}\left(e_{1}\right)=\mathrm{X} & , & \mathcal{F}_{14}\left(e_{2}\right)=\left\{x_{2}\right\}
\end{array}
$$

Hence the family of all soft- $\delta^{*}$-closed sets of the soft-tritopological space $\left(X_{v} \tau_{1}, \tau_{2}, \tau_{a}, \mathbb{E}\right) \quad$ is, S. $\delta^{*} \cdot \mathrm{C}(X)=\left\{X_{v} \Phi_{v}\left(\mathcal{F}_{\mathrm{i}}^{\mathrm{C}}, \mathbb{E}\right)\right\}, \quad$ (with $\mathrm{i}=1_{x} 2_{v \ldots r} 14$ ), defined as;

$$
\begin{aligned}
& \mathcal{F}_{1}^{c}\left(e_{1}\right)=\left\{x_{2}\right\} \quad, \quad \mathcal{F}_{1}^{C}\left(e_{2}\right)=\left\{x_{2}\right\} \\
& \mathcal{F}_{2}^{\mathcal{C}}\left(e_{1}\right)=\left\{x_{2}\right\} \quad, \quad \mathcal{F}_{2}^{\mathcal{C}}\left(e_{2}\right)=\left\{x_{1}\right\} \\
& \mathcal{F}_{\mathrm{a}}^{\mathrm{C}}\left(e_{1}\right)=\left\{x_{2}\right\} \quad, \quad \mathcal{F}_{\mathrm{a}}^{\mathrm{C}}\left(e_{2}\right)=\emptyset \\
& \mathcal{F}_{4}^{C}\left(e_{1}\right)=\left\{x_{2}\right\} \quad, \quad F_{4}^{C}\left(e_{2}\right)=\mathrm{X} \\
& \mathcal{F}_{5}^{C}\left(e_{1}\right)=\left\{x_{1}\right\} \quad, \quad \mathcal{F}_{5}^{C}\left(e_{2}\right)=\left\{x_{2}\right\} \\
& \mathcal{F}_{6}^{c}\left(e_{1}\right)=\left\{x_{1}\right\} \quad, \quad \mathcal{F}_{6}^{C}\left(e_{2}\right)=\left\{x_{1}\right\} \\
& \mathcal{F}_{7}^{C}\left(e_{1}\right)=\left\{x_{1}\right\} \quad, \quad \mathcal{F}_{7}^{\mathscr{C}}\left(e_{2}\right)=\emptyset \\
& \mathcal{F}_{\mathrm{g}}^{\mathrm{C}}\left(e_{1}\right)=\left\{x_{1}\right\} \quad, \quad \mathcal{F}_{\mathrm{g}}^{\mathrm{C}}\left(e_{2}\right)=\mathrm{X} \\
& \mathcal{F}_{g}^{C}\left(e_{1}\right)=\mathrm{X} \quad, \quad \mathcal{F}_{9}^{C}\left(e_{2}\right)=\left\{x_{2}\right\} \\
& \mathcal{F}_{10}{ }^{C}\left(e_{1}\right)=\mathrm{X} \quad, \quad F_{10}{ }^{c}\left(e_{2}\right)=\left\{x_{1}\right\} \\
& \mathcal{F}_{11}^{C}\left(e_{1}\right)=\mathrm{X} \quad, \quad \mathcal{F}_{11}{ }^{c}\left(e_{2}\right)=\emptyset \\
& \mathcal{F}_{12}{ }^{C}\left(e_{1}\right)=\emptyset \quad, \quad \mathcal{F}_{12}{ }^{C}\left(e_{2}\right)=\mathrm{X} \\
& \mathcal{F}_{13}{ }^{c}\left(e_{1}\right)=\emptyset \quad, \quad \mathcal{F}_{1 a}{ }^{c}\left(e_{2}\right)=\left\{x_{2}\right\} \\
& \mathcal{F}_{14}{ }^{C}\left(e_{1}\right)=\emptyset \quad, \quad \mathcal{F}_{14}{ }^{C}\left(e_{2}\right)=\left\{x_{1}\right\}
\end{aligned}
$$


$\exists\left(\mathcal{F}_{1}{ }_{x}{ }_{x} \mathbb{E}\right),\left(\mathcal{F}_{14}{ }^{c} \mathbb{E}\right) \in \mathrm{S} . \delta^{*} \mathrm{C}(X)$ such that

$\left(\mathcal{F}_{1}{ }^{C} \mathbb{E}\right) \cap\left(\mathcal{F}_{14}{ }^{C} \mathbb{E}\right)=\Phi$

$\exists\left(\mathcal{F}_{5^{*}} \mathbb{E}\right),\left(\mathcal{F}_{9 v} \mathbb{E}\right) \in S_{.} \delta^{*}, \mathrm{O}(X)$ such that

$\left(\mathcal{F}_{1}{ }^{c}, \mathbb{E}\right) \subseteq\left(\mathcal{F}_{5^{x}} \mathbb{E}\right),\left(\mathcal{F}_{14}{ }^{c}, \mathbb{E}\right) \subseteq\left(\mathcal{F}_{9,} \mathbb{E}\right)$

And $\left(\mathcal{F}_{5^{x}} \mathbb{E}\right) \cap\left(\mathcal{F}_{g^{x}} \mathbb{E}\right)=\Phi$, and so on ...

Thus $\left(x_{v} \tau_{1}, \tau_{2}, \tau_{3}, \mathrm{E}\right)$ is a soft $-\delta^{*}$-normal space.

Let $x_{1}, x_{2} \in \mathrm{X}$ such that $x_{1} \neq x_{2}$

$\exists\left(\mathcal{F}_{1,} \mathbb{E}\right),\left(\mathcal{F}_{5^{*}} \mathbb{E}\right) \in \mathrm{S}_{x} \delta^{*}, \mathrm{O}(X)$ such that

$x_{1} \in\left(\mathcal{F}_{1}, \mathbb{E}\right), x_{2} \notin\left(\mathcal{F}_{10} \mathbb{E}\right)$ and

$x_{2} \in\left(\mathcal{F}_{5^{*}} \mathbb{E}\right), x_{1} \notin\left(\mathcal{F}_{5^{*}} \mathbb{E}\right)$

Thus $\left(x_{v} \tau_{1}, \tau_{2}, \tau_{g}, \mathbb{E}\right)$ is a soft $-\delta^{*}-\mathrm{I}_{1}-$ space .

Therefore $\left(x_{v} \tau_{1}, \tau_{2}, \tau_{72}, \mathbb{E}\right)$ is a soft $-\delta^{*}-\mathbb{T}_{4}-$ space.

Remark 4.10. (1) A soft $-\delta^{*}-T_{4}-$ space need not be a soft $-\delta^{*}-\mathrm{T}_{a}-$ space.

(2) If $\left(X_{v}, \tau_{10}, \tau_{2}, \tau_{72}, \mathbb{E}\right)$ is a soft $-\delta^{*}-\mathrm{T}_{4}$-space then $\left(\mathrm{X}_{0} \delta^{*}{ }^{*} \tau_{\mathrm{e}}\right)$ may not be a $\mathrm{T}_{4}$-space for every parameter $\mathrm{e} \in \mathbb{E}$, where $\delta^{*} \cdot \tau_{e}=\left\{F(\mathrm{e}) \mid\left(\mathrm{F}_{v} \mathbb{E}\right) \in \mathrm{S} . \delta^{*}{ }^{*} \mathrm{O}\left(X_{)}\right)\right\}[15]$

(3) If $\left(X_{v}, \tau_{1}, \tau_{2}, \tau_{2}, \mathbb{E}\right)$ is a soft $-\delta^{*}-\mathrm{T}_{4}-$ space and $\mathrm{Y}$ is a non-empty subset of $\mathrm{X}$ then the soft-subspace $\mathrm{T}$ need not be a soft $-\delta^{*}-\mathrm{T}_{4}-$ space.

Example 4.11. Let $\mathrm{X}=\left\{x_{1}, x_{2}, x_{2}, x_{4}\right\}$ be a universe set and $\mathbb{E}=\left\{\varepsilon_{1}, e_{2}\right\}$ be the set of parameters, then by [5], the soft- set $\left(\mathrm{X}_{v} \mathbb{E}\right)$ cardinality is $|\mathcal{P}(X)|=2^{\sum_{\omega \in} \in \mathcal{H}(\mathrm{e}) \mid}$, s.t. $|\mathcal{F}(\mathrm{e})|$ is the cardinality of $\mathcal{F}(\mathrm{e})$. ( that is mean $|\mathcal{P}(x)|=2^{\mathbb{Q}}=256$ soft - set $)$.

And let $\left(X_{v}, \tau_{1}, \mathbb{E}\right),\left(X_{v}, \tau_{2}, \mathbb{E}\right)$ and $\left(x_{v} \tau_{2}, \mathbb{E}\right)$ be the three soft-topological spaces on $X$, and the soft-topologies defined as follows:

$$
\begin{aligned}
& \tau_{1}=\left\{\Phi_{v} X_{v}\left(M_{1 v} \mathbb{E}\right)_{v, n v}\left(M_{g^{x}} \mathbb{E}\right)\right\} \\
& \tau_{2}=\left\{\Phi_{v} X_{v}\left(G_{1 v} \mathbb{E}\right)_{s, n s}\left(G_{g v} \mathbb{E}\right)\right\} \text {, and }
\end{aligned}
$$

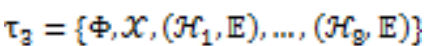

Where

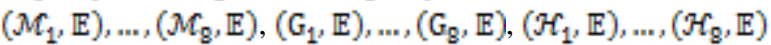
are soft-open sets in $X$, defined as follows:

$$
\begin{aligned}
& M_{1}\left(e_{1}\right)=\left\{x_{1}, x_{2}, x_{4}\right\}, \quad M_{1}\left(e_{2}\right)=\left\{x_{1}, x_{2}, x_{2}\right\} \\
& M_{2}\left(e_{1}\right)=\left\{x_{1}, x_{2}, x_{4}\right\}, \quad M_{2}\left(e_{2}\right)=\left\{x_{1}, x_{2}, x_{a}\right\} \\
& M_{a}\left(e_{1}\right)=\left\{x_{1}, x_{4}\right\} \quad, \quad M_{a}\left(e_{2}\right)=\left\{x_{1}, x_{2}, x_{a}\right\} \\
& M_{4}\left(e_{1}\right)=\left\{x_{2}, x_{2}\right\} \quad, \quad M_{4}\left(e_{2}\right)=\left\{x_{1}, x_{2}, x_{2}\right\} \\
& M_{5}\left(e_{1}\right)=\left\{x_{2}\right\} \quad, \quad M_{5}\left(e_{2}\right)=\left\{x_{1}, x_{2}, x_{2}\right\} \\
& M_{6}\left(e_{1}\right)=\left\{x_{2}\right\} \quad, \quad M_{6}\left(e_{2}\right)=\left\{x_{1}, x_{2}, x_{2}\right\} \\
& M_{7}\left(e_{1}\right)=\emptyset \quad, \quad M c_{7}\left(e_{2}\right)=\left\{x_{1}, x_{2}, x_{2}\right\} \\
& M_{\mathrm{g}}\left(e_{1}\right)=\mathrm{X} \quad, \quad M_{\mathrm{g}}\left(e_{2}\right)=\left\{x_{1}, x_{2}, x_{\mathrm{g}}\right\} \\
& \mathrm{G}_{1}\left(e_{1}\right)=\left\{x_{\mathrm{a}}\right\} \quad, \quad \mathrm{G}_{1}\left(e_{2}\right)=\left\{x_{4}\right\}
\end{aligned}
$$

$$
\begin{aligned}
& \mathrm{G}_{2}\left(e_{1}\right)=\left\{x_{2}\right\} \quad, \quad \mathrm{G}_{2}\left(e_{2}\right)=\left\{x_{4}\right\} \\
& \mathrm{G}_{\mathrm{a}}\left(e_{1}\right)=\left\{x_{2}, x_{2}\right\} \quad, \quad \mathrm{G}_{\mathrm{a}}\left(e_{2}\right)=\left\{x_{4}\right\} \\
& \mathrm{G}_{4}\left(e_{1}\right)=\left\{x_{1}, x_{4}\right\} \quad, \quad \mathrm{G}_{4}\left(e_{2}\right)=\left\{x_{4}\right\} \\
& \mathrm{G}_{5}\left(e_{1}\right)=\left\{x_{1}, x_{2}, x_{4}\right\} \quad, \quad \mathrm{G}_{5}\left(e_{2}\right)=\left\{x_{4}\right\} \\
& \mathrm{G}_{6}\left(e_{1}\right)=\left\{x_{1}, x_{2}, x_{4}\right\} \quad, \quad \mathrm{G}_{6}\left(e_{2}\right)=\left\{x_{4}\right\} \\
& \mathrm{G}_{7}\left(e_{1}\right)=\mathrm{X} \quad, \quad \mathrm{G}_{7}\left(e_{2}\right)=\left\{x_{4}\right\} \\
& \mathrm{G}_{\mathrm{g}}\left(e_{1}\right)=\emptyset \quad, \quad \mathrm{G}_{\mathrm{g}}\left(e_{2}\right)=\left\{x_{4}\right\} \\
& \mathscr{H}_{1}\left(e_{1}\right)=\left\{x_{1}, x_{2}, x_{4}\right\}, \quad \mathscr{H}_{1}\left(e_{2}\right)=\left\{x_{1}, x_{2}, x_{2}\right\} \\
& \mathscr{H}_{2}\left(e_{1}\right)=\left\{x_{1}, x_{2}, x_{4}\right\}, \quad \mathscr{H}_{2}\left(e_{2}\right)=\left\{x_{1}, x_{2}, x_{2}\right\} \\
& \mathscr{H}_{a}\left(e_{1}\right)=\left\{x_{1}, x_{4}\right\} \quad, \quad \mathscr{H}_{a}\left(e_{2}\right)=\left\{x_{1}, x_{2}, x_{a}\right\} \\
& \mathscr{H}_{4}\left(e_{1}\right)=\left\{x_{2}, x_{2}\right\} \quad, \quad \mathscr{H}_{4}\left(e_{2}\right)=\left\{x_{1}, x_{2}, x_{2}\right\} \\
& \mathscr{H}_{5}\left(e_{1}\right)=\left\{x_{2}\right\} \quad, \quad \mathscr{H}_{5}\left(e_{2}\right)=\left\{x_{1}, x_{2}, x_{2}\right\} \\
& \mathscr{H}_{6}\left(e_{1}\right)=\left\{x_{a}\right\} \quad, \quad \mathscr{H}_{6}\left(e_{2}\right)=\left\{x_{1}, x_{2}, x_{2}\right\} \\
& \mathscr{H}_{7}\left(e_{1}\right)=\emptyset \quad, \quad \mathscr{H}_{7}\left(e_{2}\right)=\left\{x_{1}, x_{2}, x_{2}\right\} \\
& \mathscr{H}_{\mathrm{g}}\left(e_{1}\right)=\mathrm{X} \quad, \quad \mathscr{H}_{\mathrm{g}}\left(e_{2}\right)=\left\{x_{1}, x_{2}, x_{\mathrm{a}}\right\}
\end{aligned}
$$

( if we examine the all 256 soft-sets), then we get the family of all soft- $\delta^{*}$-open sets of the soft-tritopological space $\left(X_{v} \tau_{1,} \tau_{2,} \tau_{\frac{7}{y}} \mathbb{E}\right)$ is,

$$
\begin{aligned}
& \left.5 . \delta^{*} \cdot \mathrm{O}(X)=\left\{X_{x} \Phi_{v}\left(\mathcal{F}_{\mathrm{i},} \mathbb{E}\right)\right\} \text {, (with } \mathrm{i}=1,2_{x} \ldots, 8\right) \\
& \mathcal{F}_{1}\left(e_{1}\right)=\left\{x_{1}, x_{2}, x_{4}\right\}, \quad \mathcal{F}_{1}\left(e_{2}\right)=\left\{x_{1}, x_{2}, x_{2}\right\} \\
& \mathcal{F}_{2}\left(e_{1}\right)=\left\{x_{1}, x_{2}, x_{4}\right\}, \quad \mathcal{F}_{2}\left(e_{2}\right)=\left\{x_{1}, x_{2}, x_{a}\right\} \\
& \mathcal{F}_{a}\left(e_{1}\right)=\left\{x_{1}, x_{4}\right\} \quad, \quad \mathcal{F}_{a}\left(e_{2}\right)=\left\{x_{1}, x_{2}, x_{a}\right\} \\
& \mathcal{F}_{4}\left(e_{1}\right)=\left\{x_{2}, x_{2}\right\} \quad, \quad \mathcal{F}_{4}\left(e_{2}\right)=\left\{x_{1}, x_{2}, x_{a}\right\} \\
& \mathcal{F}_{5}\left(e_{1}\right)=\left\{x_{2}\right\} \quad, \quad \mathcal{F}_{5}\left(e_{2}\right)=\left\{x_{1}, x_{2}, x_{2}\right\} \\
& \mathcal{F}_{6}\left(e_{1}\right)=\left\{x_{2}\right\} \quad, \quad \mathcal{F}_{6}\left(e_{2}\right)=\left\{x_{1}, x_{2}, x_{2}\right\} \\
& \mathcal{F}_{7}\left(e_{1}\right)=\emptyset \quad, \quad \mathcal{F}_{7}\left(e_{2}\right)=\left\{x_{1}, x_{2}, x_{\mathrm{a}}\right\} \\
& \mathcal{F}_{\mathrm{g}}\left(e_{1}\right)=\mathrm{X} \quad, \quad \mathcal{F}_{\mathrm{g}}\left(e_{2}\right)=\left\{x_{1}, x_{2}, x_{\mathrm{g}}\right\}
\end{aligned}
$$

We note that $\left(X_{v}, \tau_{1}, \tau_{2}, \tau_{2}, \mathbb{E}\right)$ is a soft $-\delta^{*}-\mathrm{T}_{4}-$ space but it is not a soft $-\delta^{*}-T_{3}-$ space because $x_{1} \in X$ and $\left(\mathcal{F}_{\mathrm{g}^{x}} \mathbb{E}\right)^{\mathrm{C}}$ is a soft- $\delta^{*}$-closed set in $\mathrm{X}$ such that $x_{1} \notin\left(\mathcal{F}_{\mathrm{a}}, \mathbb{E}\right)^{\mathrm{C}}$ but there do not exist soft $-\delta^{*}$ - open sets $\left(F_{v} \mathbb{E}\right)$ and $(G, \mathbb{E})$ such that $x_{1} \in\left(F_{v} \mathbb{E}\right),\left(\mathcal{F}_{a x} \mathbb{E}\right)^{\mathbb{L}} \subseteq(G, \mathbb{E})$ and $(F, \mathbb{E}) \cap\left(G_{v} \mathbb{E}\right)=\Phi$. Thus every soft $-\delta^{*}-\mathrm{T}_{4}-$ space is not necessarily a soft $-\delta^{*}-\mathrm{T}_{a}-$ space. Now we have,

$\delta^{*} \cdot \tau_{e_{2}}=\left\{\mathrm{X}_{0}, \emptyset_{s}\left\{x_{2}\right\},\left\{x_{2}\right\},\left\{x_{2}, x_{9}\right\},\left\{x_{1}, x_{4}\right\},\left\{x_{1}, x_{a}, x_{4}\right\},\left\{x_{1}, x_{2}, x_{4}\right\}\right\}$

And $\delta^{*} \cdot \tau_{\sigma_{2}}=\left\{X_{0}, \emptyset_{0}\left\{x_{1}, x_{2}, x_{2}\right\}\right\}$

Here $\left(\mathrm{X}_{v} \delta^{*} \cdot \boldsymbol{\tau}_{\sigma_{1}}\right)$ and $\left(\mathrm{X}_{v} \delta^{*} \cdot \boldsymbol{\tau}_{\sigma_{2}}\right)$ are not $\mathrm{T}_{a}$-space. This shows that if $\left(x_{v} \tau_{10}, \tau_{2}, \tau_{2 p} \mathbb{E}\right)$ is a soft $-\delta^{*}-\mathrm{T}_{4}$-space then $\left(\mathrm{X}_{v} \delta^{*}{ }^{*} \tau_{\mathrm{e}}\right)$ need not be a $\mathrm{T}_{4}$-space for every parameter $\mathrm{e} \in \mathbb{E}$.

Now, let $\mathrm{Y}=\left\{x_{1}, x_{2}, x_{2}\right\} \subseteq \mathrm{X}$. By the definition 2.14, the soft-sub sets $\left(\mathcal{F}_{\mathrm{iY},} \mathbb{E}\right)$ (with $\hat{\mathrm{i}}=1_{x} 2_{v, \ldots} 8$ ) of the 
soft $-\delta^{*}-$ open $\left(\mathcal{F}_{\mathrm{i}^{x}} \mathbb{E}\right)$ over $\mathrm{Y}$, results to be defined by:

$$
\begin{array}{lll}
\mathcal{F}_{1 \mathrm{Y}}\left(e_{1}\right)=\left\{x_{1}, x_{2}\right\} & , & \mathcal{F}_{1 \mathrm{Y}}\left(e_{2}\right)=\mathrm{Y} \\
\mathcal{F}_{2 \mathrm{Y}}\left(e_{1}\right)=\left\{x_{1}, x_{a}\right\} & , & \mathcal{F}_{2 \mathrm{Y}}\left(e_{2}\right)=\mathrm{Y} \\
\mathcal{F}_{\mathrm{aY}}\left(e_{1}\right)=\left\{x_{1}\right\} & , & \mathcal{F}_{\mathrm{aY}}\left(e_{2}\right)=\mathrm{Y} \\
\mathcal{F}_{4 \mathrm{Y}}\left(e_{1}\right)=\left\{x_{2}, x_{a}\right\} & , & \mathcal{F}_{4 \mathrm{Y}}\left(e_{2}\right)=\mathrm{Y} \\
\mathcal{F}_{5 \mathrm{Y}}\left(e_{1}\right)=\left\{x_{2}\right\} & , & \mathcal{F}_{5 \mathrm{Y}}\left(e_{2}\right)=\mathrm{Y} \\
\mathcal{F}_{6 \mathrm{Y}}\left(e_{1}\right)=\left\{x_{a}\right\} & , & \mathcal{F}_{6 \mathrm{Y}}\left(e_{2}\right)=\mathrm{Y} \\
\mathcal{F}_{7 \mathrm{Y}}\left(e_{1}\right)=\emptyset & , & \mathcal{F}_{7 \mathrm{Y}}\left(e_{2}\right)=\mathrm{Y} \\
\mathcal{F}_{\mathrm{gY}}\left(e_{1}\right)=\mathrm{Y} & , & \mathcal{F}_{\mathrm{gY}}\left(e_{2}\right)=\mathrm{Y}
\end{array}
$$

We note that soft-subspace $Y$ is not a soft- $\delta^{*}-T_{4}-$ space because $\left(\mathrm{F}_{\mathrm{aY}} \mathbb{E}\right)^{\mathrm{C}}$ and $\left(\mathrm{F}_{4 \mathrm{Y}_{0}} \mathbb{E}\right)^{\mathrm{C}}$ are soft $-\delta^{*}$ - closed sets in $Y$ such that $\left(\mathrm{F}_{a \mathrm{~W}} \mathbb{E}\right)^{\mathrm{C}} \cap\left(\mathrm{F}_{4 \mathrm{Y}_{s}} \mathbb{E}\right)^{\mathrm{C}}=\Phi$ but there do not exist any soft $-\delta^{*}-$ open sets $\left(F_{v} \mathbb{E}\right)$ and $\left(G_{v} \mathbb{E}\right)$ in $Y$ such that $\quad\left(F_{a \mathrm{Y}} \mathbb{E}\right)^{\mathrm{C}} \subseteq\left(\mathrm{F}_{v} \mathbb{E}\right), \quad\left(\mathrm{F}_{4 \mathrm{Y}}, \mathbb{E}\right)^{\mathrm{C}} \subseteq(\mathrm{G}, \mathbb{E}) \quad$ and $\left(F_{v} \mathbb{E}\right) \cap\left(G_{v} \mathbb{E}\right)=\Phi$

Thus a soft-subspace of a soft $-\delta^{*}-\mathrm{T}_{4}$-space may not be a soft $-\delta^{*}-\mathrm{T}_{4}-$ space.

\section{CONCLUSIONS}

In this work, we have continued the foundations of the theory of a soft-tritopological spaces to define and study the separation axioms of a soft-tritopological spaces. We defined soft $-\delta^{*}-\mathrm{T}_{0}, \mathrm{~T}_{1}, \mathrm{~T}_{2}, \mathrm{~T}_{\mathrm{a}}, \mathrm{T}_{4}$ spaces with respect to ordinary points and studied their behaviors in soft-tritopological spaces. These sof $t-\delta^{*}$-separation axioms would be useful for the growth of the set theory of soft-tritopology to solve some complex problems. We also discussed some soft-transmissible properties with respect to ordinary points. We hope that these results in this paper will help the researchers for strengthening the toolbox of soft -tritopology. In the next study, we extend the concept of soft $-\delta^{*}$-separation axioms in soft-tritopological spaces with respect to soft-points as well as ordinary.

\section{REFERENCES}

[1] D.A. Molodtsov, Soft set theory -first results, Computers and Mathematics with Applications 37 (4) (1999) 1931.

[2] P. K. Maji, A. R. Roy, R. Biswas. An application of soft sets in decision making problem. Computers \& Mathem. with Applications 44(8) (2002) 1077-1083.

[3] P.K. Maji, R. Biswas, A.R. Roy, Soft set theory, Comput. \& Math. with Applications 45(4) (2003) 555562 .

[4] M Shabir, M. Naz, On soft topological spaces, Comput. Math. Appl. 61(7) (2011) 1786-1799.

[5] N. Cağman, S. Karataş, S. Enginoglu. Soft Topology. Comput. and math. with appl., 62(1) (2011) 351-358.

[6] B. M. Ittanagi, Article: Soft Bitopological Spaces. International Journal of Computer Applications. 107(7) (2014) 1-4.

[7] A. F. Hassan, Soft Tritopological Spaces. International Journal of Computer Applications. 176(9) (2017) 26-30 .

[8] A. F. Hassan, An Introduction to Fuzzy Soft Tritopological Spaces., International Journal of Fuzzy Mathematical Archive., 17(1) (2019) 41-49.

[9] N. Giorgio., Soft N-Topological Spaces.Math.GN. 2019 May; arXiv:1905.12331 [math.GN]

[10] D. N. Georgiou, A. C. Megaritis, V. I. Petropoulos: On Soft Topological Spaces, Applied Mathematics and Information Sciences 7(2) (2013) 1889-1901.

[11] A. Kandil, , O.A Tantawy, S.A. EI- Sheikh, A.M. Abd-eLatif, Soft semi- separation axioms and irresolute soft function. FMI, 8 (2) (2014) 305-318.

[12] S.A. El-Sheikh, R.A. Hosny, A.M. Abd-e-Latif,. Characterization of $\mathrm{b}$ soft separation Axioms in soft topological spaces. Infor. Sc. Letter, 4(3) (2015) 125133.

[13] A. Khattak, G. Ali Khan, Y. Khan, M. Khattak, F. Jamal, Characterization Of Soft B-Separation Axioms In Soft Bi-Topological Spaces, Mat. Sc. Math., 2(2) (2018) 1117.

[14] M. Naz, , M. Shabir, , M.I. Ali,. Separation axiom in bi soft topological spaces. Arxiv. 1850 of $.00866 \mathrm{vl}$ [Math, Gn] 29 August (2015).

[15] A. F. Hassan, A. H. Abass. Characterization Of Soft Open Set In Soft Tritopological Space,. International scientific conference (Baghdad) . submitted.

[16] A. F. Hassan, A. H. Abass,. The Soft-Subspace of Soft-tritopological space. J. of new theory, submitted. 